\title{
Experimental Evaluation of a Mixed Controller That Amplifies Spatial Errors and Reduces Timing Errors
}

\author{
Laura Marchal-Crespo ${ }^{1,2 *}$, Tanja Baumann ${ }^{1}$, Michael Imobersteg ${ }^{1}$, Steve Maassen ${ }^{1}$ and \\ Robert Riener ${ }^{1,2}$

\begin{abstract}
${ }^{1}$ Sensory-Motor Systems (SMS) Lab, Department of Health Sciences and Technology (D-HEST), Institute of Robotics and Intelligent Systems (IRIS), ETH Zurich, Zurich, Switzerland, ${ }^{2}$ Reharobotics Group, Spinal Cord Injury Center, Balgrist University Hospital, Medical Faculty, University of Zurich, Zurich, Switzerland
\end{abstract}

\section{OPEN ACCESS}

Edited by:

Elena De Momi,

Polytechnic University of Milan, Italy

Reviewed by:

Ilana Nisky,

Ben-Gurion University of the Negev at

Beersheba, Israel

Claudia Casellato,

Polytechnic University of Milan, Italy

*Correspondence:

Laura Marchal-Crespo

laura.marchal@hest.ethz.ch

Specialty section:

This article was submitted to

Biomedical Robotics, a section of the

journal Frontiers in Robotics and Al

Received: 21 October 2016 Accepted: 05 May 2017

Published: 22 June 2017

Citation:

Marchal-Crespo L, Baumann T, Imobersteg M, Maassen S and

Riener R (2017) Experimental Evaluation of a Mixed Controller That Amplifies Spatial Errors and Reduces Timing Errors.

Front. Robot. Al 4:19. doi: 10.3389/frobt.2017.00019
Research on motor learning suggests that training with haptic guidance enhances learning of the timing components of motor tasks, whereas error amplification is better for learning the spatial components. We present a novel mixed guidance controller that combines haptic guidance and error amplification to simultaneously promote learning of the timing and spatial components of complex motor tasks. The controller is realized using a force field around the desired position. This force field has a stable manifold tangential to the trajectory that guides subjects in velocity-related aspects. The force field has an unstable manifold perpendicular to the trajectory, which amplifies the perpendicular (spatial) error. We also designed a controller that applies randomly varying, unpredictable disturbing forces to enhance the subjects' active participation by pushing them away from their "comfort zone." We conducted an experiment with thirty-two healthy subjects to evaluate the impact of four different training strategies on motor skill learning and selfreported motivation: (i) No haptics, (ii) mixed guidance, (iii) perpendicular error amplification and tangential haptic guidance provided in sequential order, and (iv) randomly varying disturbing forces. Subjects trained two motor tasks using ARMin IV, a robotic exoskeleton for upper limb rehabilitation: follow circles with an ellipsoidal speed profile, and move along a 3D line following a complex speed profile. Mixed guidance showed no detectable learning advantages over the other groups. Results suggest that the effectiveness of the training strategies depends on the subjects' initial skill level. Mixed guidance seemed to benefit subjects who performed the circle task with smaller errors during baseline (i.e., initially more skilled subjects), while training with no haptics was more beneficial for subjects who created larger errors (i.e., less skilled subjects). Therefore, perhaps the high functional difficulty of the tasks limited the potential benefit of mixed guidance. Adding random disturbing forces during training reduced the learning effect size compared to no haptics. The unanticipated forces also decreased the subjects' feelings of competence while did not increase their effort and interest. Further studies with mildly affected neurologically patients employing easier tasks need to be performed in order to evaluate the applicability of our approaches in rehabilitation.

Keywords: motor learning, haptic guidance, error amplification, random disturbing forces, rehabilitation robotics, mixed guidance controller, motivation 


\section{INTRODUCTION}

There is increasing interest in using robotic devices to improve or gain new motor skills and recover lost motor function after a neurologic injury (e.g., stroke). There has been a progression in the development of robotic training strategies that specify how these robotic devices interact with subjects-see Marchal-Crespo and Reinkensmeyer (2009) for a review. The most extended paradigm is to use a robotic device to physically guide subjects' limbs during movement training. It is thought that by moving the limb in ways that subjects would otherwise not be able to move might motivate them to engage in repetitive and intensive practice (Reinkensmeyer and Housman, 2007). However, robotic guidance also appears to decrease physical effort during training (Israel et al., 2006) suggesting that robotic training could potentially decrease motor learning and/or recovery if it encourages slacking, i.e., a decrease of muscle activation during repeated movements when movement errors are small (Scheidt et al., 2000; Reinkensmeyer et al., 2009). In a recent clinical study with chronic stroke survivors, training with guidance from an exoskeleton robot enhanced motor function more effectively than conventional therapy. However, the benefits were limited to severe impaired patients (Klamroth-Marganska et al., 2014). Thereby, current rehabilitation robots might be working with suboptimal training strategies-only using a fraction of the rehabilitation potential-by not considering the subjects' individual needs.

The goal of robotic therapy is to provoke motor plasticity in order to improve motor recovery. Currently, however, there is not a solid scientific understanding of how this goal can best be achieved. Recent work has emphasized the relevance of motor learning in neurorehabilitation (Krakauer, 2006; Shumway-Cook and Woollacott, 2007). In fact, it is commonly accepted in the field of neurorehabilitation that recovery is a form of learning or relearning (Krakauer, 2015). Understanding the underlying mechanisms of learning during the acquisition of novel motor skills may provide novel ways to improve neurorehabilitation therapies.

There is emerging evidence that haptic guidance may be specifically useful for learning timing tasks (Marchal-Crespo et al., $2015 a, b)$. Several studies have shown a benefit of haptic guidance in learning to reproduce the temporal, but not spatial, characteristics of complex spatiotemporal curves (Feygin et al., 2002; Lüttgen and Heuer, 2012). The effect of haptic guidance on learning a visuo-manual tracking task has been evaluated in several studies, which found a positive effect of guidance on the time-related components of a dynamic task, such as an increase in movement speed and smoothness (Bluteau et al., 2008) and better learning of temporal force patterns (Morris et al., 2007). Training with haptic guidance also benefited learning to play a pinball-like game, which is a highly time-critical task (Milot et al., 2010). These results suggest that haptic demonstration of optimal timing, rather than movement magnitude, may have facilitated skill transfer (Heuer and Lüttgen, 2015). Nevertheless, in a recent experiment we found that the most effective robotic training condition depended on the degree of rhythmicity of the task: haptic guidance hampered learning of continuous rhythmic tasks, but promoted learning of discrete movements (Marchal-Crespo et al., 2015a,b).
Experimental evidence has demonstrated that it is possible to accelerate learning processes in healthy subjects when trajectory errors are accentuated using robotic forces during walking (Emken and Reinkensmeyer, 2005). In patients with chronic stroke, amplifying errors during reaching with a force field resulted in straighter movements when the force field was removed (Patton et al., 2006). Similarly, enhancing errors using constant disturbing forces in children with dystonia resulted in better performance in reaching and rhythmic tasks (circle drawing) when the force was removed (Casellato et al., 2012). Increasing limb phasing error in post-stroke patients' gait through a split-belt treadmill also induced a long term increase in walking symmetry (Reisman et al., 2007). However, there are also studies that did not find a benefit from augmenting errors during training. Increasing velocity errors when learning a golf putting task had no detectable effect on task performance (Duarte and Reinkensmeyer, 2015). In fact, augmenting errors resulted in a decrease on perceived competence and satisfaction and a longterm decrease in motivation. These contradictory results suggest that error amplification might limit learning if it decreases motivation during training due to systematically performing large errors. Alternatively, researchers have proposed that error amplification might benefit learning of only some specific characteristics of the training tasks, such as spatial features of trajectories (Heuer and Lüttgen, 2015).

Movement errors can also be induced using randomly varying feedforward forces that disturb subjects' movements during training. Recent research has shown that subjects whose movements were more variable before training could more rapidly adapt to a perturbation than those with reduced variability (Wu et al., 2014). This is in line with recent research that states that error exploration-i.e., encouraging learners to examine and investigate new tasks by themselves-is an important element to boost learning, especially during the first states of learning (Huberdeau et al., 2015). Disturbing subjects with randomly varying feedforward forces could potentially improve movement variability, and therefore, result in better motor learning. Furthermore, in recent experiments, we found that adding random disturbing forces during training improved motor learning of a locomotion task, probably because the addition of unforeseen forces increased subjects' effort (muscle activation) and concentration (Marchal-Crespo et al., 2014a,b).

There is an initial body of work that compared the effectiveness of different robotic trainings that augment or reduce movement errors on motor learning (Feygin et al., 2002; Lee and Choi, 2010). However, results from these comparative studies were highly inconclusive, probably because they searched for the robotic training strategy that results in better motor learning, independently of the subjects' individual skill level and the specific characteristics of the task to be learned. Haptic guidance seemed to be particularly helpful for initially less skilled subjects (Marchal-Crespo et al., 2010a,b, 2013), while error amplification was found to be more beneficial for skilled subjects (Milot et al., 2010). The rhythmicity and spatial-temporal characteristics of the motor task to be learned also seemed to be key factors to consider when selecting the robotic training strategies that enhance motor learning (Marchal-Crespo et al., 2015a,b). 
Many everyday tasks include both temporal and spatial characteristics. For example, in dance moves, the path followed and the timing along the path are critical factors. Learning to draw a circle with a particular speed profile is a motor task with a timing component (learning the speed profile), but also with a relevant spatial component (drawing a circle) (Lüttgen and Heuer, 2012). In order to promote learning of both temporal and spatial task characteristics simultaneously, we designed a novel mixed guidance controller that concurrently combines haptic guidance and error amplification. We ran a motor learning experiment with thirty-two healthy subjects to evaluate the impact on motor learning and self-reported motivation of four different training strategies: (i) No haptics, (ii) a novel mixed guidance controller, (iii) perpendicular error amplification and tangential haptic guidance provided in random sequential order, and (iv) randomly varying unpredictable disturbing forces. The third training strategy was included to compare the relative benefits on motor learning of applying the tangential haptic guidance and perpendicular error amplification strategies simultaneously (mixed guidance), or in random sequential order. ARMin IV, a robotic exoskeleton for upper limb rehabilitation, was used to conduct the experiment. Subjects were requested to learn two different motor tasks: a rhythmic task-i.e., move the hand following circles with an ellipsoidal speed profile, and a continuous single task-i.e., move the hand along a $3 \mathrm{D}$ line following a complex speed profile. Subjects were tested in both tasks before and after training, with and without visual feedback.

We hypothesized that providing haptic guidance to learn the time components-i.e., reducing errors tangential to the desired trajectory-and error augmentation to learn the spatial components-i.e., augmenting perpendicular errors-would result in better learning of the timing and spatial characteristics. We expected better motor learning when the error amplification and haptic guidance were provided simultaneously (mixed guidance) compared to sequential order, since subjects trained in sequential order would only benefit from each training strategy half of the time. We also hypothesized that training with random disturbing forces would decrease feelings of competence but would increase subjects' effort during training, resulting in better learning. Since the mixed guidance strategy employed both, haptic guidance and error amplification simultaneously, we did not expect it to have a great effect on motivation.

\section{MATERIALS AND METHODS}

\section{ARMin IV}

We employed ARMin IV (Figure 1) to conduct the motor learning experiment. ARMin IV is a 7 DoF robotic device for upper limb rehabilitation designed by the SMS-lab at ETH Zurich in collaboration with the University Hospital Balgrist (Nef et al., 2009). The robot incorporates position and force/torque sensors to measure the interaction between human and robot which enable subjects to perform a wide range of natural movements of the upper extremities (Guidali et al., 2011). The spatial precision of the measurements is less than $0.5 \mathrm{~cm}$. A real time system with a sampling rate of $1 \mathrm{kHz}$ controls the device and communicates with the virtual world over a UDP connection.

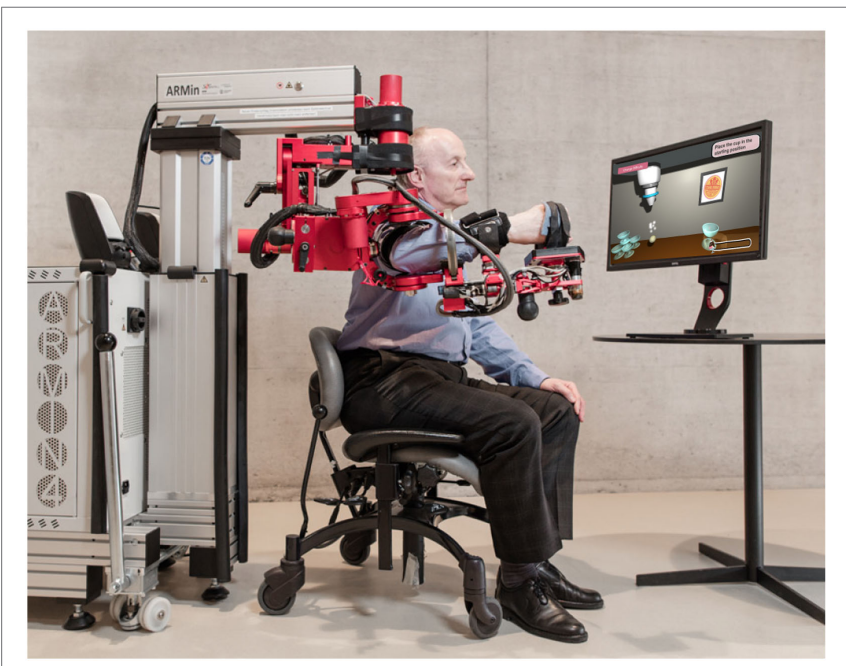

FIGURE 1 | ARMin IV is a 7 DoF robotic device for upper limb rehabilitation that incorporates position and force/torque sensors (Guidali et al., 2011).

\section{Training Strategies}

\section{No Haptics}

Compensating for undesired robot dynamics is critical in order to allow the participants to perform the tasks by themselves. Ideally, the robot should be transparent, i.e., the interaction torques between robot and patient should be zero. We designed the no haptics controller to minimize the interaction torques using a closed-loop zero-torque controller. However, due to the mass and friction of the exoskeleton, transparency cannot be fully achieved through torque control. Therefore, the force of gravity acting on the exoskeleton (Nef et al., 2009) and the forces due to friction of the motor-gear combinations were modeled and used as feedforward compensation (Guidali et al., 2011). Remaining inertia effects, however, could not be compensated, since the robot did not have acceleration sensors and the second derivative of the position signal was noisy and could have led to instability. However, the motor tasks to be learned were quite slow and therefore, the robot felt very transparent.

\section{Tangential Haptic Guidance}

In order to assist subjects to learn the time components of the task, we developed a proportional controller to reduce errors tangential to the desired trajectory. This controller works as a force field that is linked to the desired position and is, therefore, time dependent (Figure 2A). The force field has a stable manifold tangential to the trajectory, which acts as a haptic guidance controller, guiding the subjects in velocity-related aspects. The forces exerted by the tangential haptic guidance controller $\left(F_{t}\right)$ can be described as:

$$
F_{t}=k_{t} \cdot\left(x_{d t}-x\right)=k_{t} \cdot e_{t} ; \text { where } k_{t}>0 \text {. }
$$

Equation 1 describes a position controller in end-effector coordinates where the error in tangential direction $\left(\boldsymbol{e}_{t}\right)$ is reduced with a positive proportional gain $k_{t}$. The perpendicular and tangential directions are defined by the tangent with respect to the trajectory going through the desired position $\left(x_{d}\right)$ at a certain time step 

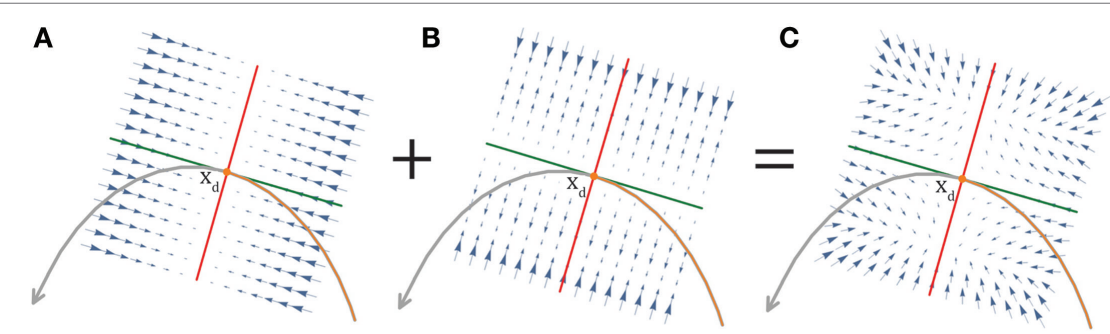

FIGURE 2 | Example of force field generated by: (A) the tangential haptic guidance controller at a desired position ( $\left.x_{d}\right)$ on the target trajectory, (B) the perpendicular error amplification controller with tubular transition to haptic guidance for large errors, and (C) the tubular mixed guidance controller.

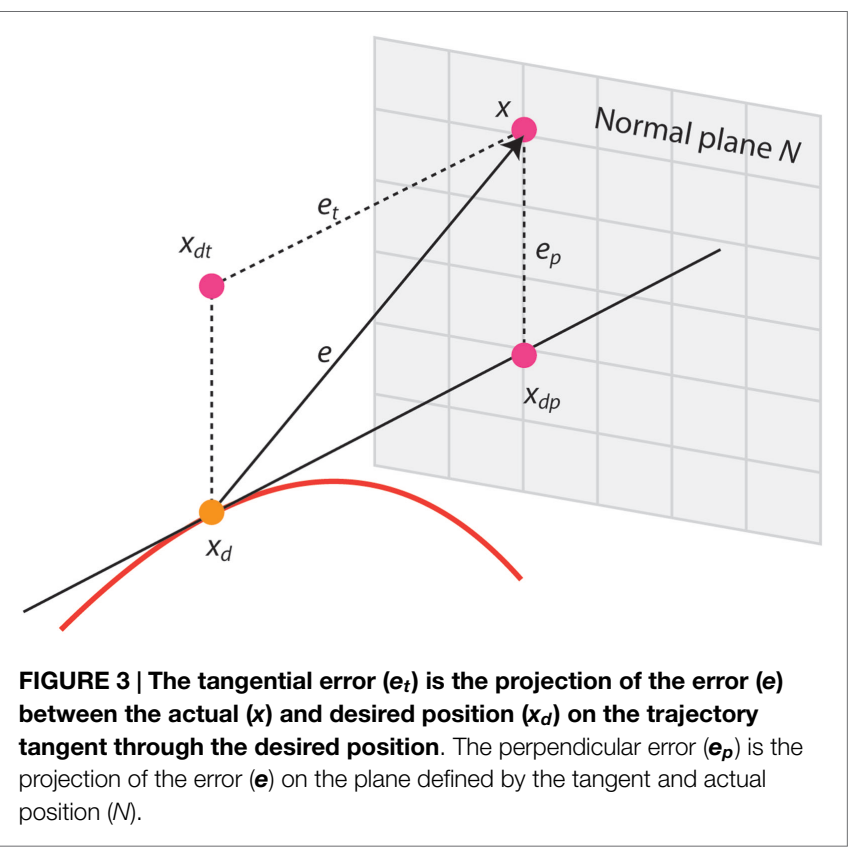

(Figure 3). The tangential error $\left(e_{t}\right)$ is the projection of the error $(e)$ between the actual $(x)$ and desired position $\left(x_{d}\right)$ on the trajectory tangent through the desired position. The perpendicular error $\left(e_{p}\right)$ is the projection of the error $(e)$ on the normal plane $(N)$ defined by the tangent and actual position.

We entirely waived any derivative gains from the controller in order to keep the force feedback as intuitive and predictable as possible, and therefore, facilitate the building of an internal model. The proportional gain $k_{t}$ was chosen ad hoc. In order to guide the subjects in velocity-related aspects, they should be guided accurately through time to convey a feeling of the right timing. This can only be accomplished with a stiff position controller that suppresses all tangential errors. Since gravity increases the difficulty of any task in vertical direction, additional assistance needed to be provided to compensate for the subject's arm weight. These restrictions resulted in gains of $10 \mathrm{~N} / \mathrm{cm}$ in the $x$ and $y$ directions, and $20 \mathrm{~N} / \mathrm{cm}$ in the vertical direction $(z)$.

The haptic force in end-effector coordinates was saturated at $10 \mathrm{~N}$ to guarantee subjects' safety, then transformed into joint torques by using inverse kinematics and input to a closed-loop torque controller (impedance controller), where friction and weight compensation were applied.

\section{Perpendicular Error Amplification}

In order to augment the spatial errors, we developed a proportional controller to amplify errors perpendicular to the desired trajectory. This controller works as a force field linked to the desired position that has an unstable manifold perpendicular to the trajectory (Figure 2B), which acts as an error amplification controller. The forces exerted by the perpendicular error amplification controller $\left(F_{p}\right)$ can be described as:

$$
F_{p}=k_{p} \cdot\left(x_{d p}-x\right)=k_{p} \cdot e_{p} ; \text { where } k_{p}<0 .
$$

Equation 2 describes a position controller in end-effector coordinates where the error in the perpendicular direction $\left(e_{p}\right)$ is amplified with a negative proportional gain $k_{p}$. The error amplification controller leads to an unstable system, i.e., repulsive forces increase proportionally to perpendicular errors. Considering that subjects have a maximum force that they can apply, coming back to the desired trajectory would be impossible if the amplification forces increase linearly with the error. In order to limit large errors that can be dangerous or discouraging for the participants, error amplification should be applied only in a small predefined area around the desired position $\left(\boldsymbol{x}_{\boldsymbol{d}}\right)$. By additionally realizing a conversion toward haptic guidance when the error is too large, the subject could further be supported upon poor performance.

We made the negative proportional gain $k_{p}$ a function of the subject's ongoing perpendicular error in order to limit dangerous and discouraging large errors with haptic guidance, while augmenting awareness of task relevant errors by means of error amplification (Rüdt et al., 2016). The used equation corresponds to the superposition of two elementary sigmoid functions:

$$
k_{p}=K_{\max } \cdot\left(\frac{2}{1+\exp \left(r \cdot\left(\left|e_{p}\right|-e_{\mathrm{turn}}\right)\right)}-1\right) .
$$

The resulting function can be tuned by three parameters. The parameter $K_{\max }$ stands for the maximal amplification factor when the perpendicular error $e_{p}$ is equal to zero. The parameter $e_{\text {turn }}$ defines the error where the gain $k_{p}$ changes its sign (Figure 4). Due to this change, the amplification force also changes its sign, transitioning from error amplification to haptic guidance. The combined sigmoid function has saturation for small and large $e_{p}$. The width of the saturated area and the steepness of the curve around $e_{\text {turn }}$ can be tuned using the parameter $r$.

This approach resulted in a three-dimensional tube-like structure wrapped around the current tangent (Figure 2B). Within this 

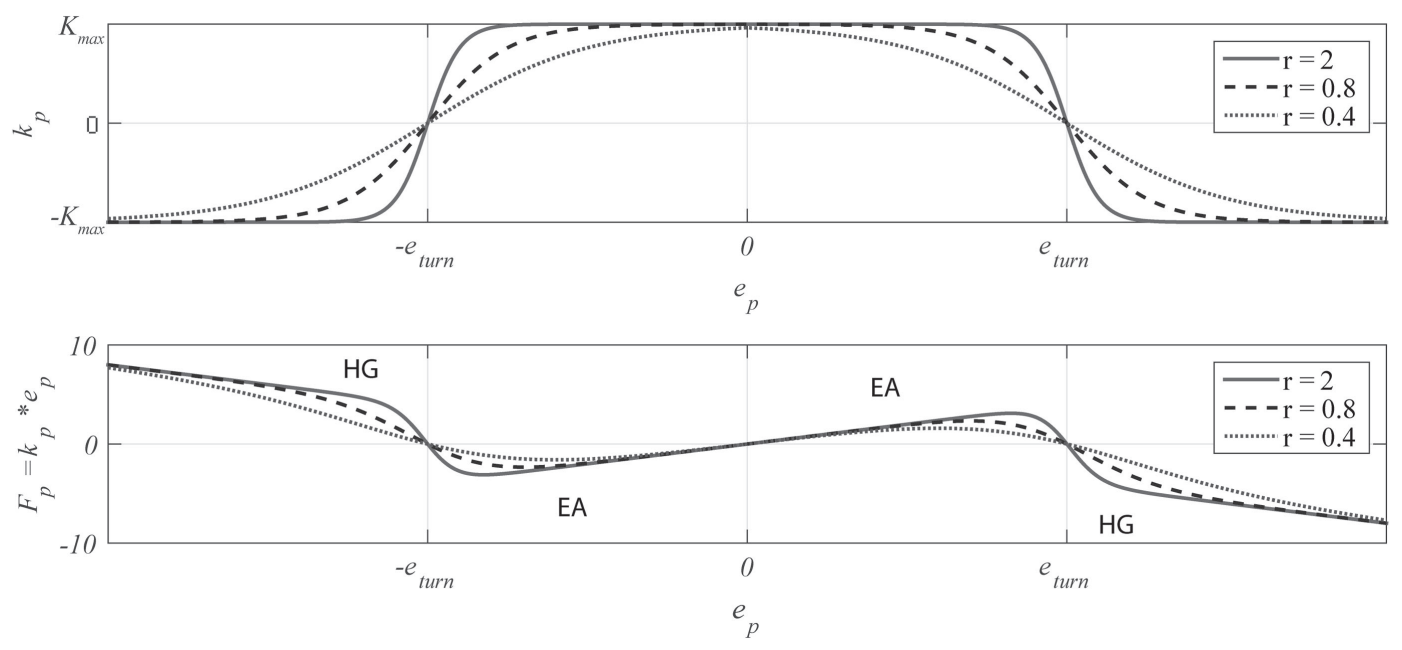

FIGURE 4 | Combined sigmoid function and its resulting perpendicular force. The proportional gain $k_{p}$ is function of the subject's ongoing perpendicular error, limits dangerous and discouraging large errors (errors larger than $e_{\text {turn }}$ ) with haptic guidance $(\mathrm{HG})$, while augmenting awareness of task relevant errors by means of error amplification (EA).

tube, error amplification was applied. Once the subject leaved this surrounding, haptic guidance would be employed to limit errors larger than $e_{\text {turn }}$.

The maximal amplification factor $K_{\max }$ was chosen ad hoc. Contrary to the tangential haptic guidance controller, the perpendicular error amplification approach should allow subjects to make small errors in order to stimulate motor learning. Nevertheless, subjects should be able to also stay on the desired target position. In order to enable a certain tracking accuracy without an excessive physical effort from the subject, we chose a small $K_{\max }$ gain of $0.4 \mathrm{~N} / \mathrm{cm}$. In order to provide a good compromise between smooth transition and unmodified control modes, we set the slope $r$ of the sigmoid to 0.8 .

Finally, the perpendicular force in end-effector coordinates was saturated at $10 \mathrm{~N}$ to guarantee subjects' safety, transformed into joint torques and input to a closed-loop torque controller.

\section{Mixed Guidance with Tubular Transition}

In order to enhance learning of both, temporal and spatial task characteristics simultaneously, we designed a novel mixed guidance controller that combined the tangential haptic guidance and the perpendicular error amplification controllers described above. The mixed guidance controller is realized by a force field with a stable manifold tangential to the trajectory, which acts as a haptic guidance controller and an unstable manifold perpendicular to the trajectory that acts as an error augmentation controller (Figure 2C). To implement the mixed guidance approach with tangential haptic guidance and perpendicular error amplification, the two sets of control forces derived in equations 1 and 3 were superimposed:

$\boldsymbol{F}=k_{p} \cdot\left(x_{d p}-\boldsymbol{x}\right)+k_{t} \cdot\left(x_{d t}-\boldsymbol{x}\right)=k_{p} \cdot \boldsymbol{e}_{p}+k_{t} \cdot \boldsymbol{e}_{t} ;$ where $k_{p}\left\langle 0, k_{t}\right\rangle 0$.

We made the negative proportional gain $k_{p}$ a function of the subject's ongoing perpendicular error in order to limit large errors with haptic guidance, while augmenting task relevant errors with error amplification using equation 3. This fusion resulted in a tubular structure surrounding the current trajectory tangent. Within this tube, the mixed guidance approach remained unchanged. However, once the perpendicular errors got bigger than $e_{\text {turn }}$, haptic guidance was applied in all dimensions. To ensure the safety and directionality of the resulting forces, the control output got saturated to $10 \mathrm{~N}$ only after superposition. The total force calculated in end-effector coordinates by the controller was then transformed into joint torques by using inverse kinematics, and fed into a closed-loop torque controller.

\section{Random Disturbing Forces}

The idea of the randomly varying disturbing forces controller is to enhance the active participation and concentration of subjects by pushing them away from their "comfort zone." The implementation of the random disturbing force controller with ARMin IV is specified by some key parameters that describe the forces' directionality, frequency and magnitude.

For the directional aspect, the reference system in which the random disturbing forces are applied plays a crucial role (i.e., joint or end-effector space). For the current application (learning a trajectory in end-effector space), we chose end-effector coordinates for consistency. In order to have the random disturbing forces only acting on the spatial aspect of the task, the disturbing forces were generated so that they were randomly allocated parallel to the trajectory normal plane $(N)$ at each sample time (Figure 3).

The timing aspect is tuned by three parameters. First, the average time between two pulses $\left(T_{\mathrm{avg}}\right)$ is specified. This parameter defines the average frequency of application of random disturbing forces. Second, the duration of a disturbance $\left(T_{\text {dist }}\right)$ is fixed. Third, the minimal time between two pulses $\left(T_{\min }\right)$ is chosen. This parameter controls that there is a minimum idle time between two consecutive disturbances to allow subjects to recover from the previous unforeseen force. 
The timing condition for the random disturbing forces can be formulated applying probability calculation. At every time step, a random number rand in the range from zero to one is drawn from a uniform distribution [rand $\sim \operatorname{Unif}(0,1)]$. The probability for this random number rand to be smaller than a constant $C \in[0,1]$ is equal to $C$ (Rüdt et al., 2016).

$$
\operatorname{Pr}(\text { rand }<C)=C \text {. }
$$

If rand is smaller than $C$, a disturbance is applied. Since this process runs at $1 \mathrm{kHz}$, disturbances are applied at an average frequency $\left(f_{\text {avg }}\right)$ of $1,000 \mathrm{C} \mathrm{Hz}$.

The magnitude of the disturbances is drawn from a uniform distribution in the range between the minimal desired disturbance force $\left(F_{\text {distmin }}\right)$ and the maximal desired force $\left(F_{\text {distmax }}\right)$ :

$$
F_{\text {dist }}=F_{\text {distmin }}+\text { rand } \times\left(F_{\text {distmax }}-F_{\text {distmin }}\right) .
$$

All timing and magnitude parameters were selected ad hoc. The average time $T_{\text {avg }}$ was set to $4 \mathrm{~s}$ in order to keep the subjects' attention on one hand, but also prevent frustration. The duration of a pulse $T_{\text {dist }}$ was set to $0.4 \mathrm{~s}$. This duration sufficed for force application by the robotic device, but was still perceived as discrete disturbance. The minimal time between two pulses $\left(T_{\min }\right)$ was fixed at $1 \mathrm{~s}$. The thresholds for the minimum and maximum exerted forces $\left(F_{\text {distmin }}\right.$ and $\left.F_{\text {distmax }}\right)$ were set to 10 and $20 \mathrm{~N}$, respectively. Although these values seem relatively large, the remaining moment of inertia of the robot hampered their effect on the final subjects' movements, since the unanticipated forces had the effect of a short and fast change in the movement's smoothness (Marchal-Crespo et al., 2011).

\section{The Ice Cream Maker Game}

We developed a virtual game, the Ice Cream Maker, using Unity@5.0 (Unity Technologies, USA). In this game, the subject takes on the role of an ice cream vendor, who needs to make an ice cream for a young customer. The aim of the game was to serve ice cream by placing a cup under a dispenser that followed predefined trajectories (Figure 5). Under the dispenser, the desired cup position was indicated with a white sparkling particle effect that moved with the dispenser. The location of the cup in the game corresponds to the location of the end-effector of the robot in the real world. The white sparkles turned green whenever the cup was held in the correct position. This visual cue was included to help subjects locate the cup in $3 \mathrm{D}$ space within the $2 \mathrm{D}$ game view.

Two dispensers (one for ice cream and one for toppings) moved following two different trajectories. In previous studies we found that the training strategy that benefits motor learning the most depends on the specific characteristics of the task to be learned. In particular, the rhythmicity and duration of the movement seemed to play a key role on the effectiveness of robotic training (MarchalCrespo et al., 2015a,b). Therefore, we selected two different movements-usually trained during clinical practice-that differed in their rhythmicity.

\section{Rhythmic Trajectory}

The ice cream dispenser followed continuous and consecutive circles on the horizontal plane with an ellipsoidal speed profile (Figures 5A,C) (Lüttgen and Heuer, 2012). Tracking a circle is a task usually employed in robot-assisted rehabilitation during training and assessment (Dipietro et al., 2007; Nordin et al., 2014).

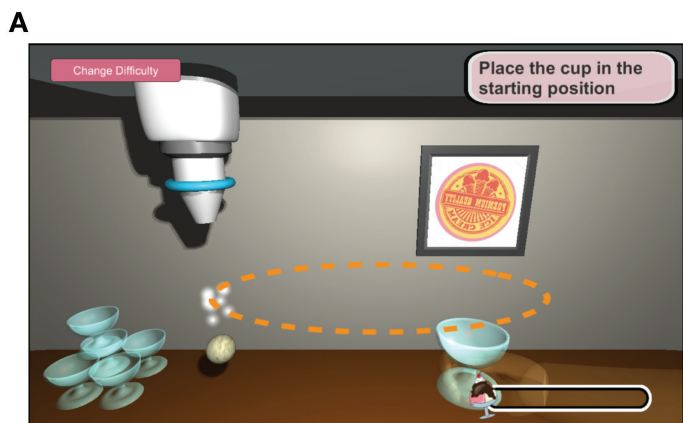

C Desired Velocity Profile in Circle

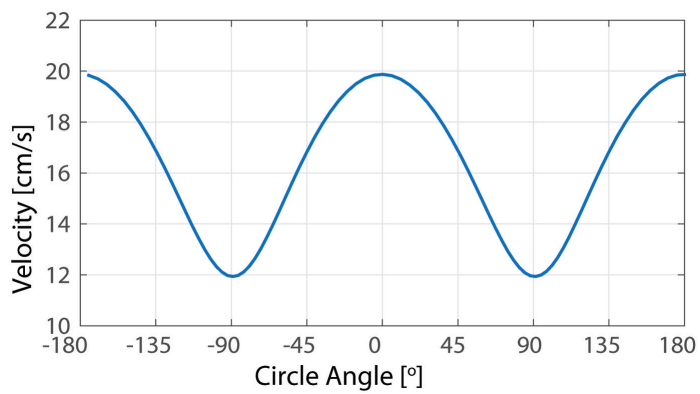

B

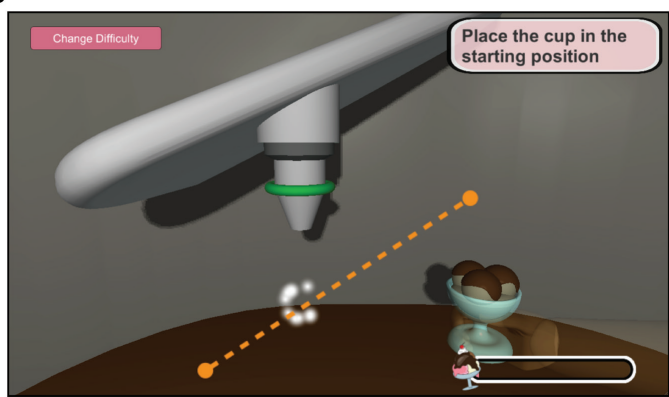

D Desired Velocity Profile in Line

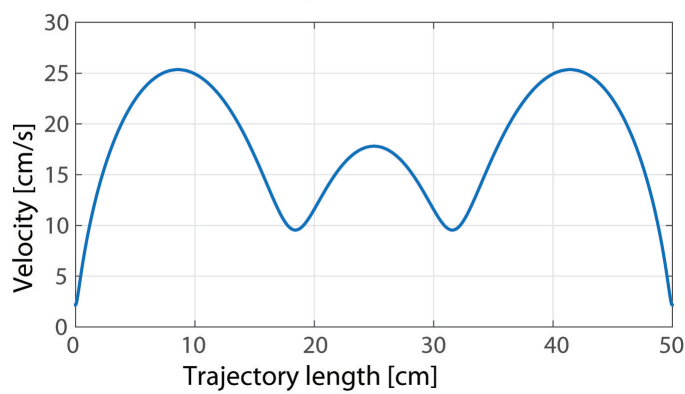

FIGURE 5 | (A) Screen shot of the ice cream dispenser (circle). (B) Screen shot of the toppings dispenser (line). (C) Desired speed profile in the circle task. (D) Desired speed profile in the line task. The dashed lines were not displayed to subjects. 
The circle's radius was set to $10.6 \mathrm{~cm}$ and the maximum and minimum speeds were 20 and $12 \mathrm{~cm} / \mathrm{s}$, respectively.

\section{Single Trajectory}

The toppings dispenser moved along the estimated line of proprioceptive neuromuscular facilitation, i.e., a line that spans $50 \mathrm{~cm}$ from the bottom left to the upper right in $3 \mathrm{D}$. This is an effective movement regularly used in physiotherapy to increase joint range of motion (Kwak and Ryu, 2015). The desired speed profile consisted of the combination of three Gaussians, two with peak speeds of $25.3 \mathrm{~cm} / \mathrm{s}$ and one in the middle with peak speed $17.8 \mathrm{~cm} / \mathrm{s}$ (Figures 5B,D). The toppings dropped on the two local minima and at the end of the desired velocity profile. A 3-s counting down message was presented on the screen as a cue to inform subjects that the line movement was about to begin.

We artificially created the desired velocity profiles in order to increase the task difficulty and to add a strong timing component into the tasks. Since the experiment was performed with healthy subjects, performing the trajectories at their natural velocity profiles (i.e., bell-shaped velocity profile in the line and constant velocity in the circle) would have been too easy.

\section{Experimental Protocol}

The study was approved by the local ethical committee (Kantonale Ethikkommission Zürich, Application Number: KEK-ZH-Nr. 2015-0013) and conducted in compliance with the Declaration of Helsinki. Thirty-two healthy, right-handed adult subjects (14 female), $25.8 \pm 3.6$ years old, gave written consent to participate in the study.

Subjects were randomly assigned to one of four training strategies (eight subjects per group): No haptics $(\mathrm{NH})$, mixed guidance (MG), tangential haptic guidance + perpendicular error amplification provided in sequential order $(\mathrm{HG}+\mathrm{EA})$, and random disturbing forces $(\mathrm{RF})$. The HG + EA group was included in the study in order to compare the relative benefits on motor learning of applying the tangential haptic guidance and perpendicular error amplification strategies simultaneously (mixed guidance), or in sequential order. The eight subjects in the HG + EA were further randomly assigned into two groups. The first group (four subjects) started training with tangential haptic guidance and finished with perpendicular error amplification, while the second group (four subjects) started with error amplification and finished with haptic guidance.

Subjects were instructed to learn the two different movements (circle and line) followed by the dispenser. In order to prevent a possible influence of the task order, subjects in each group were randomly assigned into two groups, which learned the tasks in an opposite order.

The experiment took place in two different days (Figure 6). On day 1 , subjects started with a baseline test $(1 \mathrm{~min})$ where they were instructed to track the dispenser (that followed the line or the circle, depending on the task order) in order to fill the cup without guidance/disturbance from the robot. During baseline free ( $1 \mathrm{~min})$, subjects were requested to reproduce the movements
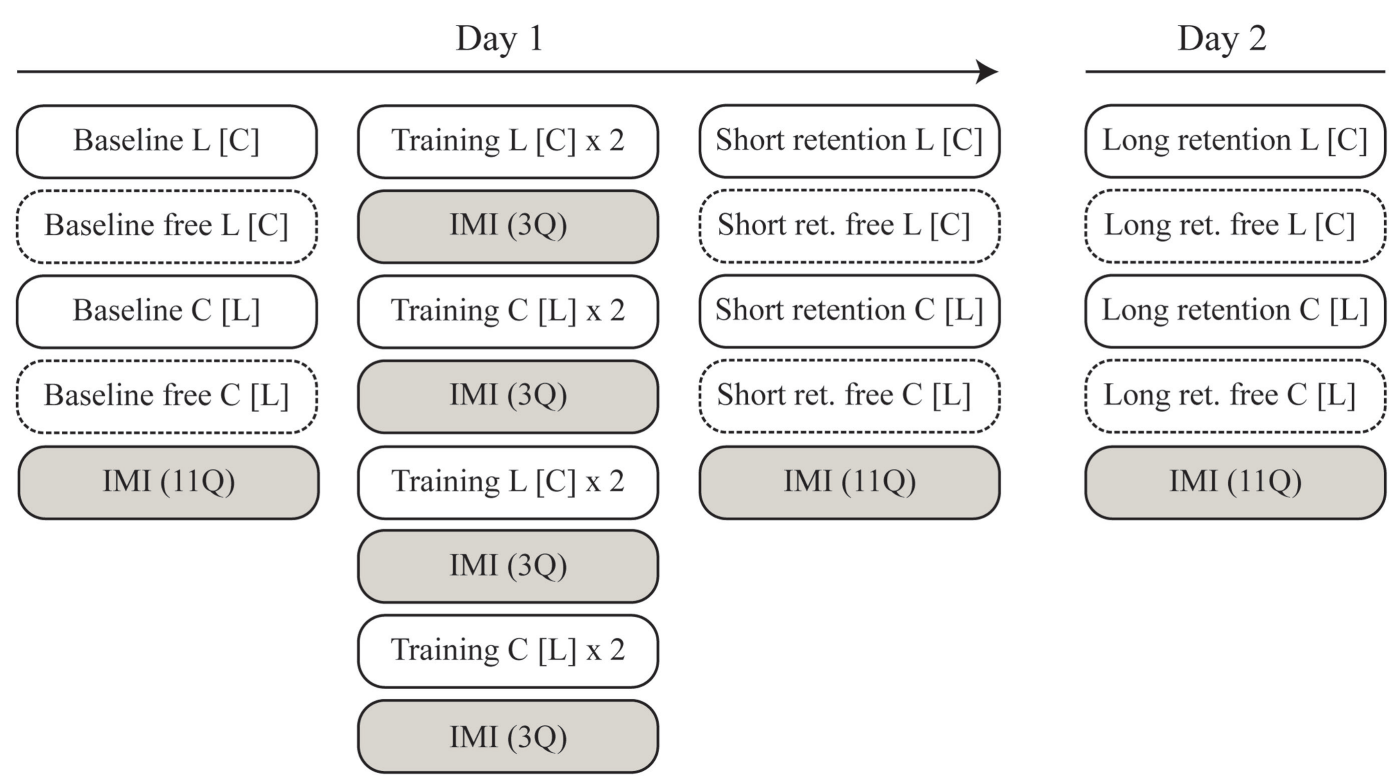

FIGURE 6 | Experimental protocol. On day 1, subjects started with a baseline test (1 min) where they were instructed to track the dispenser that followed the line $(\mathrm{L})$ or the circle $(\mathrm{C})$, depending on the order of the task. During baseline free (1 min), subjects were asked to reproduce the movement without visual feedback (dashed blocks). Baseline and baseline free were then repeated for the second task. During the training blocks, subjects trained with no haptics, mixed guidance, $\mathrm{HG}+\mathrm{EA}$, or random disturbing forces, depending on their training group, in eight blocks of 1.5 min each. The order of the tasks to be trained was alternated. First, subjects performed two training blocks with the first task, followed by two blocks with the second task. These four blocks were then repeated again. After a 5 -min break, short-term retention and short-term retention free tests per each task were performed that followed the same structure and order than the baseline and baseline free tests. After 3-5 days subjects came back to perform the long-term retention and long-term retention free tests. After the second baseline free test, and at the end of both experimental days, subjects responded to 11 questions selected from the Intrinsic Motivation Inventory (IMI). Subjects also responded to the first three questions of the questionnaire after every two training blocks. 
they just performed during baseline, but without visual feedback, i.e., the dispenser did not move. Baseline and baseline free tests were then repeated for the second task. During the training blocks, subjects trained with no haptics, mixed guidance, $\mathrm{HG}+\mathrm{EA}$ or random disturbing forces, depending on their training strategy group, in eight blocks of $1.5 \mathrm{~min}$ each. We alternated the order of the tasks to be trained. First, subjects performed two training blocks with the first task (the first task in baseline), followed by two blocks with the second task. These four blocks were then repeated again. After a 5-min break, subjects performed shortterm retention and short-term retention free tests per each task that followed the same structure and order than the baseline and baseline free tests. The total experiment time on the first day was around $1 \mathrm{~h}$. Subjects were invited to return after 3-5 days to perform the long-term retention and long-term retention free tests, which followed the same structure and order as the short-term retention and short-term retention free tests. Short rest breaks every two training blocks were allowed to minimize subjects' fatigue.

After the second baseline free test, and at the end of both experimental days, subjects responded to 11 questions (Table 1) selected from the Intrinsic Motivation Inventory (IMI, Ryan, 1982), which is a well-established and valid questionnaire already used in previous motor learning and rehabilitation studies (Novak et al., 2014; Duarte and Reinkensmeyer, 2015). The full questionnaire consists of 45 questions and can be further divided into 7 subscales. In the present study, only four subscales were selected: interest/enjoyment (I/E), perceived competence (PC), effort/importance $(\mathrm{E} / \mathrm{I})$, and pressure/tension $(\mathrm{P} / \mathrm{T})$. These four items were selected because they encompass the essentials of the motivational aspects in the specific motor task (McAuley et al., 1989). Subjects also responded to the first three questions of the questionnaire (Table 1, in bold) after every two training blocks (Figure 6). We selected these questions in order to assess subjects' enjoyment, perceived competence and effort during training. Subjects rated their feelings on a Likert scale between 1 and 7 points; 1 indicated "I disagree completely" and 7 indicated "I agree completely." The questions were presented in German and

TABLE 1 | IMI Questionnaire.

\begin{tabular}{|c|c|}
\hline IMI Questions & Subscale \\
\hline 1. I enjoyed playing the game very much & interest/enjoyment (I/E) \\
\hline $\begin{array}{l}\text { 2. I am satisfied with my performance } \\
\text { in the game }\end{array}$ & $\begin{array}{l}\text { perceived } \\
\text { competence (PC) }\end{array}$ \\
\hline 3. I put a lot of effort into this & effort/importance (E/I) \\
\hline 4. I think the game was boring & $(\mathrm{I} / \mathrm{E})$ \\
\hline 5. I felt very tense while playing the game & pressure/tension $(\mathrm{P} / \mathrm{T})$ \\
\hline 6. I could not play the game very well & $(\mathrm{PC})$ \\
\hline 7. It was important for me to do well at the game & $(\mathrm{E} / \mathrm{l})$ \\
\hline 8. This game was fun to play & $(\mathrm{l} / \mathrm{E})$ \\
\hline 9. I was anxious while playing the game & $(\mathrm{P} / \mathrm{T})$ \\
\hline 10. I think I am pretty good at the game & $(\mathrm{PC})$ \\
\hline 11. I did not try very hard to do well at the game & $(\mathrm{E} / \mathrm{l})$ \\
\hline
\end{tabular}

Set of 11 questions selected from the Intrinsic Motivation Inventory (IMI) (Ryan, 1982) in order to assess subjects' interest/enjoyment (I/E), perceived competence (PC), effort/importance (E/I) and pressure/tension (P/T). Questions in bold were also presented after each two training blocks. Subjects rated on a Likert scale between 1 and 7 points; 1 indicated "I disagree completely" and 7 indicated "I agree completely."
English. Subjects could see their previous answers to the same questions.

\section{Data Processing and Statistical Analysis}

We calculated the mean absolute tracking error (i.e., the mean absolute difference between the measured and desired position), its perpendicular and tangential projections, and the mean absolute velocity error (i.e., the mean absolute difference between the measured and desired velocity) during baseline, training and short- and long-term retention tests. We evaluated the performance during baseline free and short- and long-term retention free tests (i.e., when subjects were requested to reproduce the task without visual feedback) comparing the recorded and desired trajectories using dynamic time warping (DTW) with the weighting of the temporal shifts set to zero (i.e., spatial error) (Giese and Poggio, 2000). We also used DTW to calculate the velocity error, i.e., comparing the recorded and desired velocity profiles using DTW with the weighting of the temporal shifts set to zero. Data from one subject in the random disturbing force group (RF) and one subject in the no haptics group $(\mathrm{NH})$ while practicing the line task were discarded because they did not follow the instructions despite coaching, and instead systematically performed very large errors. After removing these subjects, data became normal distributed during baseline for each group.

We used repeated measures of ANOVA to test the effect that different training strategies, task to be learned and initial skill level had on the error reduction from baseline to retention tests. The error created during baseline was used as a qualitative measure of initial skill level (i.e., the larger the error during baseline, the initially less skilled a subject was). We fitted the model with the two different tasks (circle vs. line, fixed effect), the four different training strategies [no haptics $(\mathrm{NH})$, mixed guidance (MG), tangential haptic guidance + perpendicular error amplification in series $(H G+E A)$, and random disturbing forces $(R F)$ as fixed effect], the initial skill level as covariate, and the subjects as random effect. We evaluated the main effects of the training strategy, the task to be learned and the initial skill level and the interaction between the training strategy and task. We performed Pearson's correlation tests in order to test the correlation between error reduction after the different training strategies and initial skill level (i.e., performance during baseline).

We used multivariate analysis in order to test whether the training strategies modulated subjects' performance during training. We evaluated the performance change from baseline to the first training block with the four training strategies and the two tasks to be learned as fixed effects. We performed univariate analysis with the performance change from baseline to the first training block with the training strategy as fixed effect in order to evaluate the influence of the training strategy on performance for the line and the circle tasks. We note that during the first training block, half the subjects in the HG + EA group trained with tangential haptic guidance, while the other half trained with perpendicular error amplification.

In order to measure the effects that the different training strategies had on self-reported motivation, we compared the responses to the 11 IMI questions just before the training started and after 
short- and long-term retention tests. We grouped the responses into each of the four subscales: interest/enjoyment (Q1, Q4, Q8), perceived competence (Q2, Q6, Q10), effort/importance (Q3, Q7, $\mathrm{Q} 11)$ and pressure/tension (Q5, Q9). We also analyzed the effect of the different training strategies on self-reported motivation during training using the subset of three questions from the IMI given after every two consecutive training blocks (four short IMI questionnaires in total). We used the responses before training as the reference point. We compared the changes in responses using the non-parametric independent samples Kruskal-Wallis test with training strategy as the main factor. Mann-Whitney test range test was used on pairwise comparisons, if the Kruskal-Wallis was significant. We used Wilcoxon signed-ranks tests to compare changes in the responses from baseline to short- and long-term retention tests.

We checked normal distribution visually using Q-Q plots. We performed post hoc comparisons with Bonferroni correction. When Mauchly's test indicated that the assumption of sphericity was violated for repeated measures ANOVA, we used Greenhouse-Geisser corrections and adjusted degrees of freedom were reported. The significance value was set to $\alpha=0.05$. We also report $p$-values smaller than 0.1 as tendencies. Statistical analyses were performed using $\mathrm{IBM}^{\circledast}$ SPSS $^{\circledast}$ Software (version 21, Chicago, IL, USA).

\section{RESULTS}

\section{Performance during Training with Different Training Strategies}

The robotic guidance provided in the tangential direction during training with mixed guidance and $\mathrm{HG}+\mathrm{EA}$ reduced the tangential errors when performing the line and circle tasks. We found a significant effect of training strategy between baseline and first training block on the tangential error [Figure 7A, $F(3,53)=3.01, p=0.038]$. Contrast revealed that subjects who trained with mixed guidance reduced the tangential error to a greater percentage than subjects who practiced with random disturbing forces (Figure 7A, $p=0.013$ ) and tended to reduce the error more than subjects who practiced without haptics (Figure 7A, $p=0.083$ ). The $\mathrm{HG}+\mathrm{EA}$ group also reduced the tangential error in a greater amount than the random disturbing forces group (Figure 7A, $p=0.032$ ). We did not find a main effect of task; neither the interaction effect of the strategy and task was significant.

We also found a main effect of training strategy on the perpendicular error reduction between baseline and the first training block [Figure $7 \mathbf{B}, F(3,53)=4.70, p=0.006$ ]. In particular, post hoc test revealed that subjects trained without haptics reduced the perpendicular error in a greater amount than subjects in the mixed guidance group (Figure $7 \mathbf{B}, p=0.006$ ). They also reduced the error in a greater amount than subjects in the $\mathrm{HG}+\mathrm{EA}$ group, but the difference did not reach significance (Figure 7B, $p=0.096$ ). The main effect of task was non-significant $[F(1,53)=3.66, p=0.061]$. The task and strategy interaction effect was also non-significant.

The controllers' output forces reached the $10 \mathrm{~N}$ saturation limit during training with the mixed guidance controller on average $29.04 \%$ of the time and with the tangential haptic guidance controller $27.45 \%$ of the time. The gain of the error amplification controller was relatively small, and therefore, the forces from the error amplification controller never reached saturation. During training, subjects in the no haptics group reached perpendicular errors equal or greater than $e_{\text {turn }}$ on average $0.05 \%$ of the time, subjects in the mixed guidance $1.5 \%$ of the time, subjects in the random disturbing forces group $0.34 \%$, subject training with haptic guidance $0.71 \%$ and subjects during training with error amplification $0.4 \%$ of the time.

\section{Effect of Training Strategies and Tasks on Skill Learning}

We analyzed the effect that the different training strategies and tasks had on the absolute tracking error reduction from baseline to
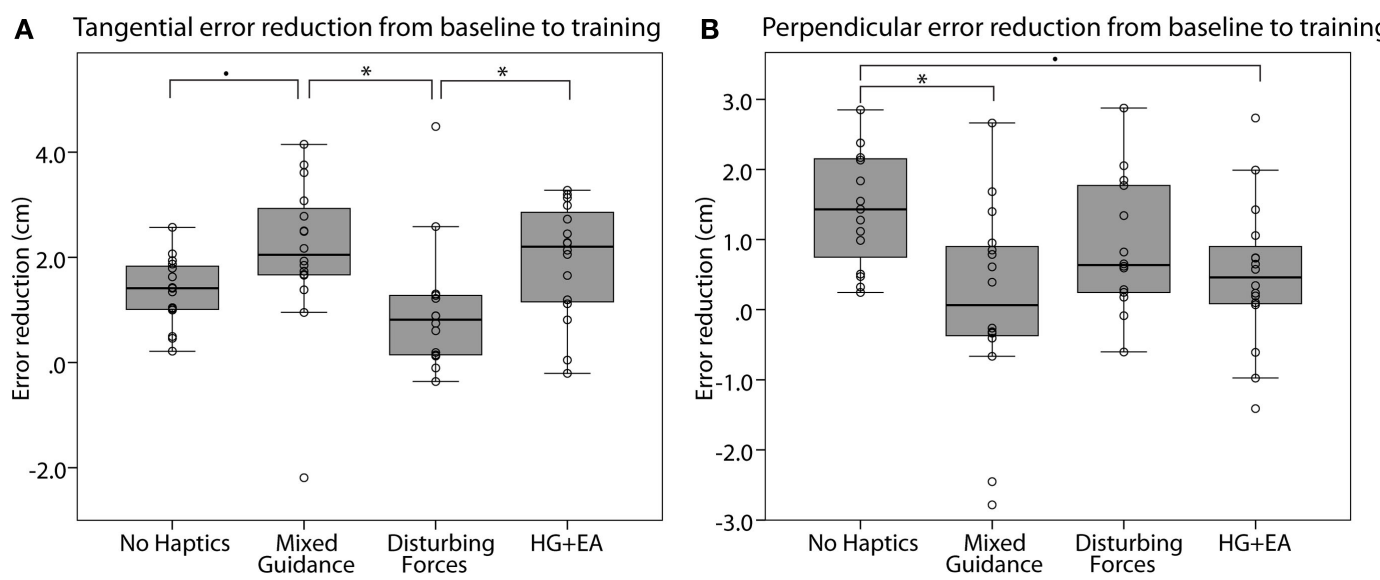

FIGURE 7 | Box-plot of error reduction from baseline to the first training block. (A) The robotic guidance provided in the tangential direction during training with mixed guidance and $\mathrm{HG}+\mathrm{EA}$ reduced the mean absolute tangential errors in a greater amount than the no haptics and random disturbing forces. (B) The error amplification provided in the perpendicular direction during training with mixed guidance and $\mathrm{HG}+\mathrm{EA}$ reduced the mean absolute perpendicular errors in a significant smaller amount than the no haptics and random disturbing forces. ${ }^{*} p<0.05, p<0.1$. Horizontal lines are medians, boxes show the interquartile range (IQR), error bars extent $\pm 1.5 \mathrm{IQR}$. Individual data points are plotted as circles. 
short-and long-term retention tests. The absolute tracking error during baseline (initial skill level) was a strong regressor on the error reduction $[F(2,106)=59.86, p<0.001]$, i.e., subjects who performed worse during baseline (initially less skilled) reduced the tracking error in a greater amount than initially more skilled subjects. We found a significant main effect of training strategy $[F(6,106)=3.034, p=0.009]$. Contrast revealed that the effect of the training strategy was significant in the absolute tracking error reduction from baseline to long-term retention [Figure 8A, $F(3,53)=3.701, p=0.017]$. In particular, post hoc tests showed than subjects in the no haptics group reduced the error to longterm retention significantly more than subjects in the random disturbing forces group (Figure 8A, $p=0.015$ ). We also found a significant effect of the task on the tracking error reduction [Figure 8A, $F(2,106)=4.14, p=0.019]$. Contrast revealed that subjects created larger tracking errors when tracking the line compared to the circle $(p=0.017)$ and that subjects reduced the error in the circle task in a greater amount than in the line task. This indicates that performing the line was more difficult than the circle. The interaction effect between task and training strategy was non-significant.

We did not find a significant main effect of the training strategy on the velocity error reduction from baseline to short- and long-term retention tests. We found that the error reduction was significantly larger when performing the circle task compared to the line task [Figure 8B, $F(2,106)=28.67, p<0.001$ ]. Contrast

\section{A Absolute tracking error reduction from baseline to long term retention}

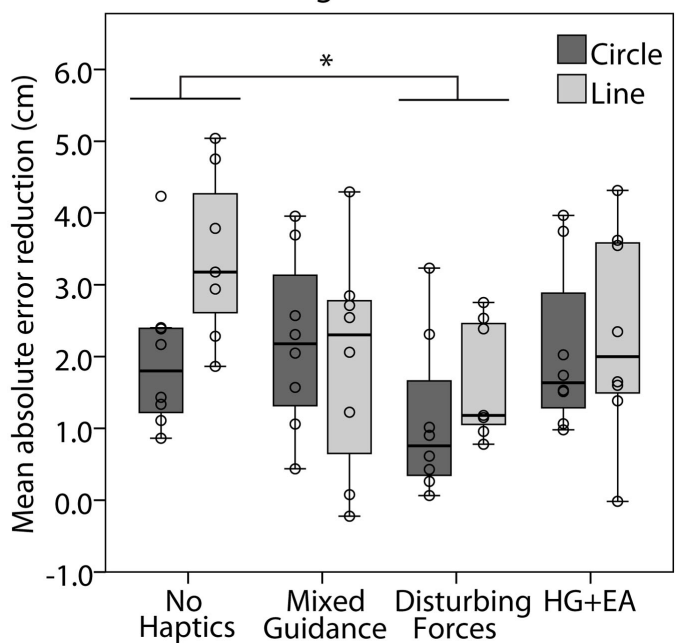

C Absolute tangential error reduction from baseline to short term retention

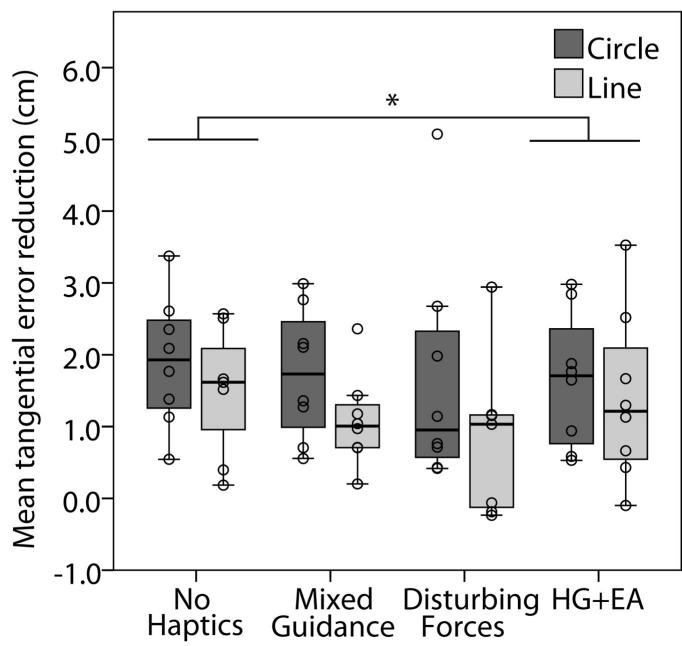

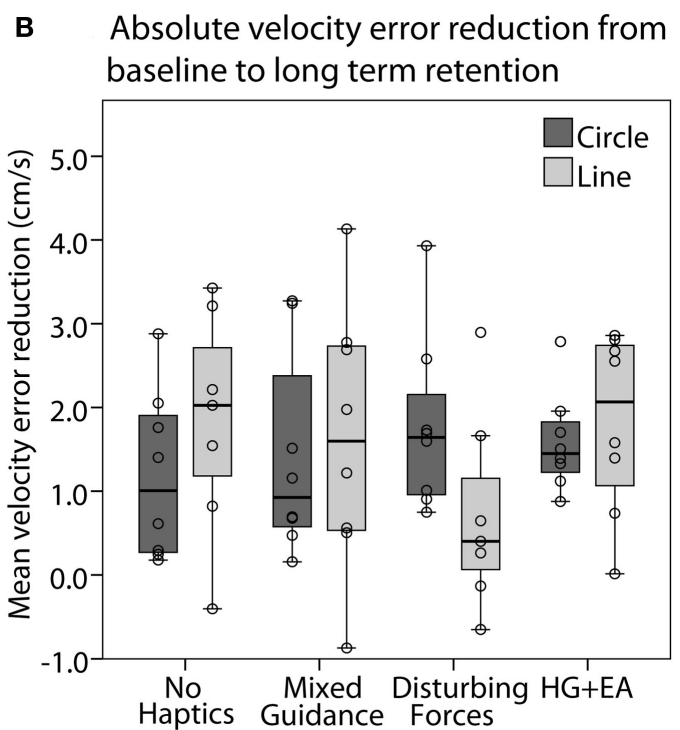

D Absolute perpendicular error reduction from baseline to long term retention

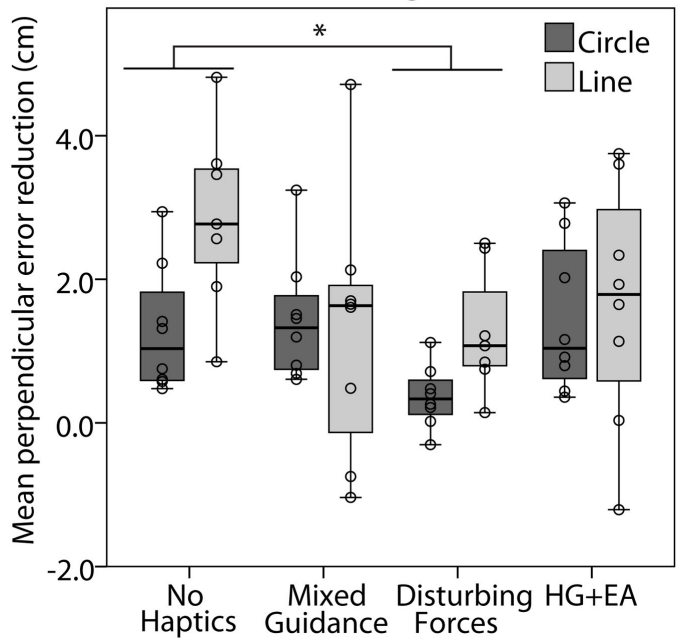

FIGURE 8 | Effect of training strategies on learning. (A) Box-plot of mean absolute tracking error reduction from baseline to long-term retention for the circle (dark gray) and line (light gray) tasks. (B) Box-plot of mean absolute velocity error reduction from baseline to long-term retention, in the circle and line tasks. (C) Box-plot of mean absolute tangential error reduction from baseline to short-term retention, in the circle and line tasks. (D) Box-plot of mean absolute perpendicular error reduction from baseline to long-term retention, in the circle and line tasks. ${ }^{\star} p<0.05$. Horizontal lines are medians, boxes show the interquartile range (IQR), error bars extent $\pm 1.5 \mathrm{IQR}$. Individual data points are plotted as circles. 
also revealed that the error during the circle task was significantly smaller than during the line task $(p<0.001)$, indicating that performing the velocity profile in the line task was more difficult than the velocity profile in the circle. The absolute velocity error during baseline was, again, a strong regressor on the error reduction $[F(2,106)=42.728, p<0.001]$. The interaction effect between task and training strategy was non-significant.

The main effect of the training strategy on the tangential error reduction after training showed a tendency $[F(6,106)=1.844$, $p=0.098]$. In particular, subjects in the no haptics group reduced the tangential error to short-term retention significantly more than subjects in the $\mathrm{HG}+\mathrm{EA}$ group (Figure 8C, $p=0.007$ ). We found a significant effect of the task on the error reduction [Figure 8C, $F(2,106)=15.87, p<0.001]$. Contrast revealed that the tangential error was larger in the line task compared to the circle task $(p<0.001)$ and that subjects reduced the error in the circle task in a greater amount than in the line task. The tangential error during baseline (initial skill level) was again a strong regressor $[F(2,106)=105.18, p<0.001]$.

The main effect of training strategy on the perpendicular error reduction showed a tendency $[F(6,106)=1.937, p=0.082]$. In particular, subjects in the no haptics group reduced the error to long-term retention significantly more than the random disturbing forces group (Figure 8D, $p=0.048$ ). There was no effect of the performed task on the error reduction, neither an interaction between task and strategy. The perpendicular error during baseline was again a strong regressor $[F(2,106)=30.27, p<0.001]$.

We further investigated the effect of the initial skill level (e.g., the perpendicular error during baseline) on the effectiveness of the different training strategies (i.e., reduction of perpendicular error from baseline to short-term retention) per each task and found that there was a significant linear relationship between initial skill level and the error reduction from baseline to retention after training with the four different training strategies in the circle task (Figure 9A, Pearson's correlation; no haptics: $R=0.888, p=0.003$; mixed guidance: $R=0.784, p=0.021$; random disturbing forces: $R=0.892, p=0.003 ; \mathrm{HG}+\mathrm{EA}$ : $R=0.682, p=0.063)$ and in two over four training strategies in the line task (Figure 9B, Pearson's correlations; no haptics: $R=0.861, p=0.013$; mixed guidance: $R=0.717, p=0.045$; random disturbing forces: $R=0.118, p=0.801 ; \mathrm{HG}+\mathrm{EA}$ : $R=0.481, p=0.228)$. The tendency in the differences between slopes $[F(3,27)=2.38, p=0.092]$ suggests that when training the circle (easy task) no haptics benefited the initially less-skilled subjects, while training with mixed guidance was more beneficial for initially more skilled (Figure 9A). On the other hand, when training the line (difficult task), training without haptics benefited all subjects, skilled and non-skilled (Figure 9B). The absolute tracking error follows the same results pattern.

\section{Effect of Training Strategies on the Performance without Visual Feedback}

In order to evaluate whether subjects learned how to perform the circle and line tasks without visual feedback, we analyzed the reduction of DTW spatial and velocity errors from baseline free to short- and long-retention free tests. Two data sets during baseline free and two during long-term retention tests could not be used, because subjects performed the circle task turning counterclockwise, while they should have turned clockwise.

In general, all subjects reduced significantly the spatial error from baseline free to short- and long-term retention free $[F(2,98)=11.614, p<0.001]$. However, we did not find a main effect of the training strategy. We found a main effect of the task $[F(2,98)=5.500, p=0.005]$. In particular, subjects performed systematically worse when playing the line compared to the circle task $(p<0.001)$, but reduced the spatial errors in greater amount in the line task.

Similarly, all subjects reduced the velocity error from baseline free to short- and long-term retention free $[F(1.66,81.18)=4.57$, $p=0.018$, Greenhouse-Geisser corrected]. We found a significant effect of the training strategy [Figure 10, $F(4.97,81.18)=3.13$,
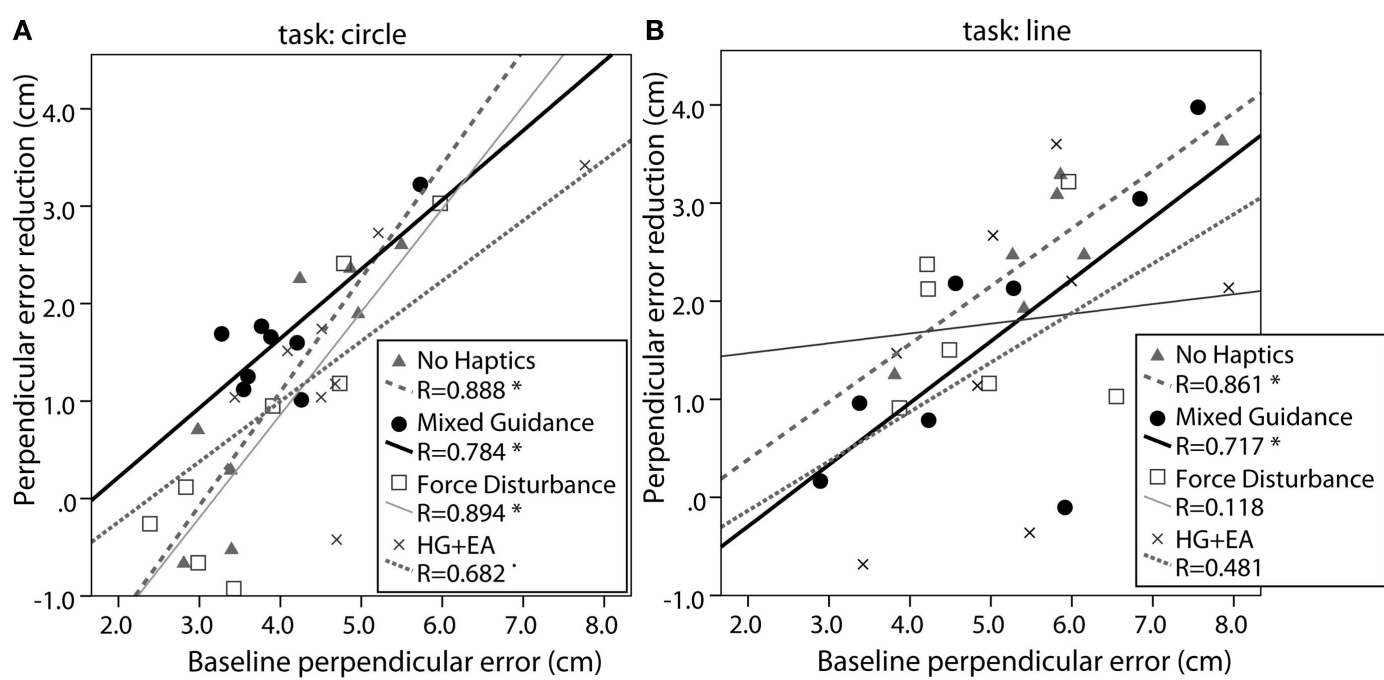

FIGURE 9 | Effect of initial skill level (baseline perpendicular error) on the perpendicular error reduction from baseline to short-term retention after training with the different training strategies for the circle task (A) and line task (B). ${ }^{*} p<0.05, p<0.1$. 
$p=0.013$, Greenhouse-Geisser corrected]. Post hoc test revealed that the velocity error reduction from baseline free to short-term retention free was smaller in the random disturbing forces group than in the no haptics $(p=0.020)$, and mixed guidance groups $(p=0.042)$. The velocity error reduction from baseline free to long-term retention free was smaller in the random disturbing forces group than in the mixed guidance group $(p=0.035)$. The effect of the task did not reach significance $[F(1.66,81.18)=2.35$, $p=0.111$, Greenhouse-Geisser corrected]. Again, the velocity error in the line was greater than in the circle $(p<0.001)$. We did not find a significant interaction between the training strategy and the task.

\section{Effect of Training Strategies on Motivation}

Training with the different strategies did not have a great impact on subjects' self-reported motivation during training

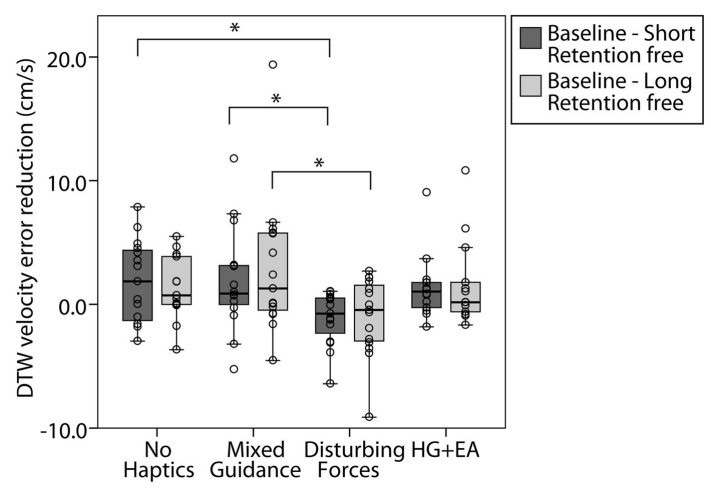

FIGURE 10 | Effect of training strategy on performance without visual feedback. Box-plot of mean DTW velocity reduction from baseline free to short-term retention free (dark gray) and to long-term retention free (light gray). ${ }^{*} p<0.05$. Horizontal lines are medians, boxes show the interquartile range (IQR), error bars extent $\pm 1.5 \mathrm{IQR}$. Individual data points are plotted as circles. (shaded areas in Figure 11). We found that, in general, subjects significantly increased the perceived competence from baseline till the end of training (Break 4) (Figure 11B, $p<0.001$ ). In particular all training groups increased significantly the perceived competence from baseline to Break 4 (no haptics, $p=0.017$; mixed guidance, $p=0.04$; HG $+\mathrm{EA}, p=0.017)$. Only subjects trained with the random disturbing forces did not significantly increase their perceived competence after the last training block. However, differences between training groups were not significant. No significant differences in perceived competence, neither on effort/importance (Figure 11C) was found between groups during training. Only the mixed guidance group showed an almost significant smaller enjoyment than the other training groups after the second training block (Figure 11A, $p=0.058$ ).

In general, subjects significantly increased their perceived competence from baseline to the end of day 1 [mean(Q2, Q6, Q10), $p<0.001]$. They also showed a non-significant decrease in effort/importance [mean(Q3, Q7, Q11), $p=0.135]$ and in pressure/tension [mean(Q5, Q9), $p=0.120]$. Subjects continued to report an increase of perceived competence after 3-5 days, when they were back for the long-term retention test $(p<0.001)$ and a reduction of pressure/tension $(p<0.001)$. In particular, all training groups-except for subjects trained with random disturbing forces-increased their perceived competence at short-term retention (Figure 12A, no haptics: $p=0.025$; mixed guidance: $p=0.028 ; \mathrm{HG}+\mathrm{EA}: p=0.012$ ). At long-term retention, also subjects in the random disturbing forces group significantly increased their perceived competence. At long-term retention, only subjects in the mixed guidance group did not significantly reduce the pressure/tension (Figure 12B).

We did not find significant differences between training groups in any of the motivation subscales. The mixed guidance group reported feeling more tense than subjects in no haptics and $\mathrm{HG}+\mathrm{EA}$ groups at short-term retention, as observed in the responses to Q5 ("I felt very tense while playing the game"). However, differences did not reach significance $(p=0.069)$. Subjects in
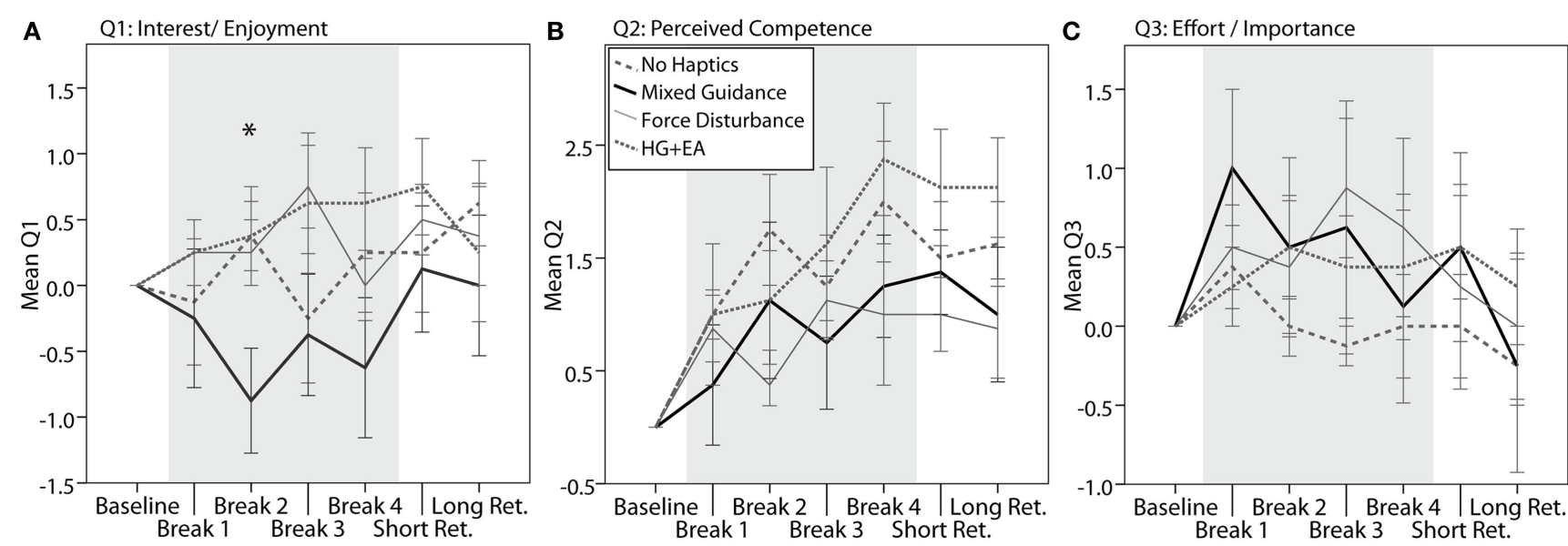

FIGURE 11 | Effect of the training strategies on self-reported motivation. (A) Responses to Q1 ("I enjoyed playing the game very much"), after baseline, after every two training blocks, and after short- and long-term retention tests. (B) Responses to Q2 ("I am satisfied with my performance in the game"). (C) Responses to Q3 ("I put a lot of effort into this"). The shaded areas indicate when the training strategies were applied. All values are referred to the responses provided after baseline. ${ }^{*} p<0.05$. Error bars: 1 SE. 

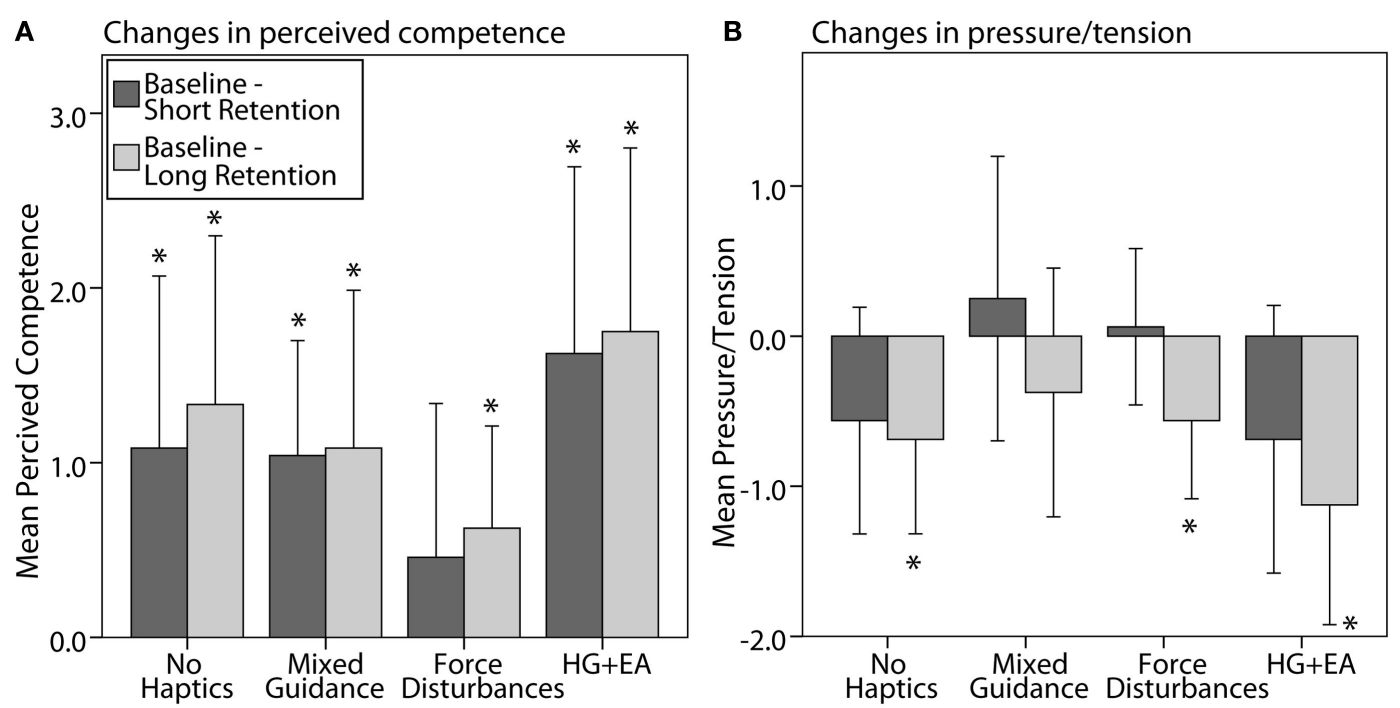

FIGURE 12 | Change in responses at short-term retention (dark gray) and after 3-5 days, at long-term retention (light gray). (A) Mean responses to Q2, Q6, and Q10 (Table 1), related to perceived competence. (B) Mean responses to Q3, Q7, and Q11 (Table 1) related to tension and pressure. All values are referred to the responses provided after baseline. ${ }^{\star} p<0.05$. Error bars: $95 \% \mathrm{Cl}$.

the random disturbing forces group seemed not to increase their perceived competence as much as subjects in the other groups, as observed in a non-significant difference in the responses to Q6 at long-term retention ("I could not play the game very well") $(p=0.105)$.

\section{DISCUSSION}

We assessed the impact of four different training strategies on motor skill learning and self-reported motivation using a robotic exoskeleton for upper limb rehabilitation: (i) no haptics: no robot forces were present, (ii) a novel mixed guidance controller: a force field with a stable manifold tangential to the desired trajectory that guides subjects in velocity-related aspects and an unstable manifold perpendicular to the trajectory, which amplifies the perpendicular (spatial) error, (iii) tangential haptic guidance and perpendicular error amplification provided in random sequential order, and (iv) random disturbing forces. Thirty-two healthy subjects were requested to learn two different motor tasks: a rhythmic task-i.e., move the hand following circles with and ellipsoidal speed profile (Lüttgen and Heuer, 2012), and a single task-i.e., move the hand along the line of proprioceptive neuromuscular facilitation (Kwak and Ryu, 2015) following a complex speed profile. Mixed guidance showed no detectable learning advantages over the other groups. However, results suggest that the effectiveness of the training strategies depends on the subjects' initial skill level.

Research in motor learning has suggested that error amplification might benefit learning of only specific characteristics of the training tasks, such as spatial features of trajectories (Casellato et al., 2012; Heuer and Lüttgen, 2015), while there is emerging evidence that haptic guidance may be specifically useful for learning reproduce the temporal, but not spatial, characteristics of trajectories (Feygin et al., 2002; Marchal-Crespo et al., 2010a,b). Thus, we hypothesized that providing haptic guidance to learn the time components-i.e., reducing errors tangential to the desired trajectory-and error augmentation to learn the spatial components-i.e., augmenting perpendicular errors-would result in better learning of timing and spatial characteristics. We expected better motor learning when the error amplification and haptic guidance were provided simultaneously (mixed guidance) than in sequential order, since subjects trained in sequential order would only benefit from each training strategy half of the time.

All training strategies worked as expected. The mixed guidance and tangential haptic guidance controllers significantly reduced the tangential tracking error when training the circle and line tasks in a greater extent than the no haptics and random disturbing forces groups. Furthermore, training with mixed guidance and perpendicular error amplification hampered the error reduction from baseline to training. We also found that performing the line task (single trajectory) was more difficult than performing the circle task (rhythmic trajectory), as suggested by the larger error created during the line task, and the smaller error reduction after training.

Contrary to our hypothesis, we found that training with mixed guidance did not result in better learning than training without haptics and training with perpendicular error amplification and tangential haptic guidance provided in sequential order. Perhaps the mixed guidance training strategy was too difficult to interpret. Error augmentation coupled with haptic guidance might not be distinguished during these complex tasks. Furthermore, the mixed guidance controller is realized by a saddle-point force field linked to the desired position, and therefore, changes its manifold directions with time in curved trajectories (tangential and perpendicular directions changed with time). Maybe this continuous change in force direction while training made the subjects to perceive the guidance as a disturbance. We observed this limitation in a preliminary study where we evaluated training 
with a first version of the mixed guidance controller and observed that training with mixed guidance limited learning of the circle (Marchal-Crespo et al., 2016). Based on those findings we decided to reduce the unstable forces in the perpendicular direction (by reducing the perpendicular error amplification controller gain) and limit large errors by adding the haptic guidance tubular transition (i.e., the transition from error amplification to haptic guidance when the perpendicular error was larger than the allowed limit). However, maybe these new additions were not sufficient to make the training easier.

Additionally, maybe the tasks to be learned were too difficult for this type of error augmenting controller. Performing circles with an ellipsoidal speed profile without arm gravity compensation was a difficult and tiring task, as suggested by the large errors done during baseline. Performing the line in 3D was even more difficult, as suggested by the larger errors created during the line task. We also observed that subjects self-reported levels of perceived competence increased significantly during training, implying that subjects did not report reaching learning ceiling effects during training, which would have been an indicator of low functional task difficulty. The reported effort after baseline was also considerable high $(5.18 \pm 1.04)$. We investigated the effect of the initial skill level on the effectiveness of the different training strategies per each task. Results suggest that when training the circle (easier task) no haptics benefited the initially less-skilled participants, while training with mixed guidance was more beneficial for initially more skilled subjects. On the other hand, when training the line (more difficult task), training without haptics benefited all subjects, independently of their initial skill level. Therefore, the unexpected limitation of the mixed guidance controller might originate from the fact that the tasks to be learned were too difficult (Marchal-Crespo et al., 2015a,b). This is in line with previous studies that showed that error amplification seemed to be particularly helpful for initially more skilled subjects (Milot et al., 2010; Marchal-Crespo et al., 2014a) and for specially simple tasks (Marchal-Crespo et al., 2014b). This can be explained by the Challenge Point Theory, which states that optimal learning is achieved when the difficulty of the task is appropriate for the individual subject's level of expertise (Guadagnoli and Lee, 2004). Thus, matching the robotic strategy-that changes the task's difficulty-to the trainee's skill level, may provide the greatest opportunity for learning.

We also found that training with perpendicular error amplification and tangential haptic guidance provided in random sequential order limited learning of the task spatial components compared to training without haptics. A possible reason is that error amplification might enhance learning only in discrete tasks, such as fast point-to-point reaching tasks (Patton et al., 2006), but might limit learning (and generalization) of tasks that require continuous error correction, such as the tracking tasks here presented. A possible rationale for the limited learning observed in our continuous tasks might originate from the feedback that subjects received while training. During training, subjects continuously received visual feedback about their performance (i.e., concurrent visual feedback). The specificity-of-learning hypothesis states that learning is most effective when training is performed involving the most crucial sensory information source needed to perform the motor task in retention tests. In our case, in order to track the ice cream dispenser, the concurrent visual information was crucial, and therefore, maybe other sources of sensory information-for example proprioception-were neglected (Proteau, 2005).

We found that adding randomly varying disturbance forces during training hampered learning of both tasks. Training with random disturbing forces resulted in poorer learning and limited performance when subjects were requested to replicate the tasks without visual feedback. A possible rationale could originate from the anticipated decrease of feelings of competence when the random disturbing forces were applied. Subjects who trained with randomly varying disturbing forces reported lower levels of competence after training. This decreased feeling of competence could have jeopardized the subjects motivation to perform the task, and therefore, negatively affect their learning outcomes (McAuley et al., 1989). This is in line with a recent study that found that robotically increasing kinematic errors did not improve learning of a golf putting task, but decreased motivation in a persistent way (Duarte and Reinkensmeyer, 2015). In a previous study, we found that the addition of random disturbing forces increased subjects effort during training (Marchal-Crespo et al., 2014b), and this increase in effort resulted in better motor learning. However, in the current study we did not find a significant increase of effort during training, probably because the task was already pretty difficult (the mean of the responses to questions related to effort was $5.18 \pm 1.04$ after baseline). Therefore, the negative effects of the decrease in perceived competence was not compensated by an increase in effort during training with random disturbing forces. Additionally, maybe the controller parameters that describe the random disturbance forces' directionality, frequency and magnitude were not appropriate to enhance motor learning of these specific tasks. We selected the parameters based on previous research on motor learning of locomotor tasks (Marchal-Crespo et al., 2014b; Rüdt et al., 2016). However, maybe complex tasks performed with the upper limbs require totally different values. Further experiments analyzing the effect of different frequency and magnitude parameters on motor learning need to be performed.

As hypothesized, since the mixed guidance strategy applied both, haptic guidance and error amplification simultaneously, it did not have a great impact on motivation. We only observed a decrease of enjoyment during the first training trials; a difference that was quickly faded as training progressed. We also observed that subjects trained with mixed guidance did not reduce the pressure/tension feelings after training, as subjects in the other training groups did. Therefore, it is still possible to apply some sort of error amplification strategy that keeps the subjects tension and pressure during training without affecting negatively their perceived competence, providing an excellent framework to enhance motor learning.

The difficulty of the motor tasks might limit the applicability of our approach in rehabilitation. The impacts of different training strategies on motor learning may differ in neurological patients (Casellato et al., 2012). They probably have a lower initial skill level and therefore, their optimal challenge point (i.e., where subjects show the best motor learning) may be at a different level. Easier tasks (e.g., drawing circles with gravity compensation) need to 
be developed and evaluated with mildly affected neurologically patients.

\section{CONCLUSION}

We evaluated a novel mixed guidance controller that combines haptic guidance and error amplification to simultaneously promote learning of the timing and spatial components of complex motor tasks. We also evaluated a controller that applies randomly varying disturbing forces to enhance the subjects' active participation by pushing them away from their "comfort zone." We found that, contrary to our hypothesis, training with mixed guidance did not result in better motor learning than training without haptics and training with error amplification and haptic guidance provided in sequential order. Results suggest that the effectiveness of the robotic training strategies depends on the subject's initial skill level, in line with our previous findings (Marchal-Crespo et al., 2015a,b). Training with mixed guidance seemed to benefit initially more skilled subjects, while training with no haptics was more beneficial for initially less skilled subjects. Therefore, the limitation of the mixed guidance strategy might be explained because the motor tasks to be learned were too difficult. Adding random disturbing forces during training showed no detectable learning advantages over no haptics. In fact, the unanticipated forces hampered learning, probably because they decreased the subjects' feelings of competence but did not increase their effort and interest. Additionally, we suggest that the benefits of error augmentation might be limited to learn tasks that require only feedforward motor control, such as point-to-point reaching tasks, but might limit learning of tasks that require continuous error correction, as is learning to track figures. An important direction for future research is to perform further studies with mildly affected neurologically patients employing easier tasks in order to evaluate the applicability of our approaches in rehabilitation.

\section{REFERENCES}

Bluteau, J., Coquillart, S., Payan, Y., and Gentaz, E. (2008). Haptic guidance improves the visuo-manual tracking of trajectories. PLoS ONE 3:e1775. doi:10.1371/journal.pone.0001775

Casellato, C., Pedrocchi, A., Zorzi, G., Rizzi, G., Ferrigno, G., and Nardocci, N. (2012). Error-enhancing robot therapy to induce motor control improvement in childhood onset primary dystonia. J. Neuroeng. Rehabil. 9, 46. doi:10.1186/ 1743-0003-9-46

Dipietro, L., Krebs, H. I., Fasoli, S. E., Volpe, B. T., Stein, J., Bever, C., et al. (2007). Changing motor synergies in chronic stroke. J. Neurophysiol. 98, 757-768. doi:10.1152/jn.01295.2006

Duarte, J. E., and Reinkensmeyer, D. J. (2015). Effects of robotically modulating kinematic variability on motor skill learning and motivation. J. Neurophysiol. 113, 2682-2691. doi:10.1152/jn.00163.2014

Emken, J. L., and Reinkensmeyer, D. J. (2005). Robot-enhanced motor learning: accelerating internal model formation during locomotion by transient dynamic amplification. IEEE Trans. Neural Syst. Rehabil. Eng. 13, 33-39. doi:10.1109/ TNSRE.2004.843173

Feygin, D., Keehner, M., and Tendick, R. (2002). "Haptic guidance: experimental evaluation of a haptic training method for a perceptual motor skill," in 10th Symposium on Haptic Interfaces for Virtual Environment and Teleoperator Systems, 2002. HAPTICS 2002 (Orlando, FL), 40-47.

Giese, M. A., and Poggio, T. (2000). Morphable models for the analysis and synthesis of complex motion patterns. Int. J. Comput. Vis. 38, 59-73. doi:10.1023/A: 1008118801668

\section{ETHICS STATEMENT}

This study was carried out in accordance with the recommendations of the Kantonale Ethikkommission Zürich (Application Number: KEK-ZH-Nr. 2015-0013) with written informed consent from all subjects. All subjects gave written informed consent in accordance with the Declaration of Helsinki. The protocol was approved by the Kantonale Ethikkommission Zürich.

\section{AUTHOR CONTRIBUTIONS}

LMC and RR contributed to the experimental design and project supervision. LMC, TB, MI and SM participated in the design of the robotic controllers and the virtual reality game. LMC and MI participated in the study design and data acquisition. LMC performed the data analysis and prepared the manuscript. All authors read and approved the final manuscript. All the authors agreed to be accountable for all aspects of the work in ensuring that questions related to the accuracy or integrity of any part of the work are appropriately investigated and resolved.

\section{ACKNOWLEDGMENTS}

The authors gratefully acknowledge the contribution of Anja Gut, Killian Baur, Jaime Duarte, Verena Klamroth-Marganska, and Fabian Just.

\section{FUNDING}

This work was partially supported by the Swiss National Science Foundation (SNF) through the grant number PMPDP2_151319 and the National Centre of Competence in Research (NCCR) Robotics.

Guadagnoli, M. A., and Lee, T. D. (2004). Challenge point: a framework for conceptualizing the effects of various practice conditions in motor learning. $J$. Mot. Behav. 36, 212-224. doi:10.3200/JMBR.36.2.212-224

Guidali, M., Duschau-Wicke, A., Broggi, S., Klamroth-Marganska, V., Nef, T., and Riener, R. (2011). A robotic system to train activities of daily living in a virtual environment. Med. Biol. Eng. Comput. 49, 1213-1223. doi:10.1007/s11517-0110809-0

Heuer, H., and Lüttgen, J. (2015). Robot assistance of motor learning: a neurocognitive perspective. Neurosci. Biobehav. Rev. 56, 222-240. doi:10.1016/j. neubiorev.2015.07.005

Huberdeau, D. M., Krakauer, J. W., and Haith, A. M. (2015). Dual-process decomposition in human sensorimotor adaptation. Curr. Opin. Neurobiol. 33, 71-77. doi:10.1016/j.conb.2015.03.003

Israel, J. F., Campbell, D. D., Kahn, J. H., and Hornby, T. G. (2006). Metabolic costs and muscle activity patterns during robotic- and therapist-assisted treadmill walking in individuals with incomplete spinal cord injury. Phys. Ther. 86, 1466-1478. doi:10.2522/ptj.20050266

Klamroth-Marganska, V., Blanco, J., Campen, K., Curt, A., Dietz, V., Ettlin, T., et al. (2014). Three-dimensional, task-specific robot therapy of the arm after stroke: a multicentre, parallel-group randomised trial. Lancet Neurol. 13, 159-166. doi:10.1016/S1474-4422(13)70305-3

Krakauer, J. W. (2006). Motor learning: its relevance to stroke recovery and neurorehabilitation. Curr. Opin. Neurol. 19, 84-90. doi:10.1097/01.wco.0000200544. 29915.cc

Krakauer, J. W. (2015). “The applicability of motor learning to neurorehabilitation," in Oxford Textbook of Neurorehabilitation, eds V. Dietz and N. Ward (Oxford 
University Press), 55-64. Available at: http://www.oxfordmedicine.com/view/ 10.1093/med/9780199673711.001.0001/med-9780199673711-chapter-7

Kwak, D. H., and Ryu, Y. U. (2015). Applying proprioceptive neuromuscular facilitation stretching: optimal contraction intensity to attain the maximum increase in range of motion in young males. J. Phys. Ther. Sci. 27, 2129-2132. doi:10.1589/jpts.27.2129

Lee, J., and Choi, S. (2010). "Effects of haptic guidance and disturbance on motor learning: potential advantage of haptic disturbance," in 2010 IEEE Haptics Symposium (Waltham, MA), 335-342.

Lüttgen, J., and Heuer, H. (2012). The influence of haptic guidance on the production of spatio-temporal patterns. Hum. Mov. Sci. 31, 519-528. doi:10.1016/j. humov.2011.07.002

Marchal-Crespo, L., Bannwart, M., Riener, R., and Vallery, H. (2015a). The effect of haptic guidance on learning a hybrid rhythmic-discrete motor task. IEEE Trans. Haptics 8, 222-234. doi:10.1109/TOH.2014.2375173

Marchal-Crespo, L., Wolf, P., Gerig, N., Rauter, G., Jaeger, L., Vallery, H., et al. (2015b). "The role of skill level and motor task characteristics on the effectiveness of robotic training: first results," in 2015 IEEE International Conference on Rehabilitation Robotics (ICORR) (Singapore), 151-156.

Marchal-Crespo, L., Baumann, T., Fichmann, D., Maassen, S., Duarte, J., and Riener, R. (2016). Evaluation of a mixed controller that amplifies spatial errors while reducing timing errors. Presented at the 38th Annual International Conference of the IEEE Engineering in Medicine and Biology Society (EMBC'16), Orlando, FL.

Marchal-Crespo, L., Furumasu, J., and Reinkensmeyer, D. J. (2010a). A robotic wheelchair trainer: design overview and a feasibility study. J. Neuroeng. Rehabil. 7, 40. doi:10.1186/1743-0003-7-40

Marchal-Crespo, L., McHughen, S., Cramer, S. C., and Reinkensmeyer, D. J. (2010b). The effect of haptic guidance, aging, and initial skill level on motor learning of a steering task. Exp. Brain Res. 201, 209-220. doi:10.1007/s00221009-2026-8

Marchal-Crespo, L., Hollnagel, C., Brügger, M., Kollias, S., and Riener, R. (2011). An fMRI pilot study to evaluate brain activation associated with locomotion adaptation. IEEE Int. Conf. Rehabil. Robot. 2011, 5975371. doi:10.1109/ICORR. 2011.5975371

Marchal-Crespo, L., López-Olóriz, J., Jaeger, L., and Riener, R. (2014a). Optimizing learning of a locomotor task: amplifying errors as needed. Conf. Proc. IEEE Eng. Med. Biol. Soc. 2014, 5304-5307. doi:10.1109/EMBC.2014.6944823

Marchal-Crespo, L., Schneider, J., Jaeger, L., and Riener, R. (2014b). Learning a locomotor task: with or without errors? J. Neuroeng. Rehabil. 11, 25. doi:10.1186/ 1743-0003-11-25

Marchal-Crespo, L., and Reinkensmeyer, D. J. (2009). Review of control strategies for robotic movement training after neurologic injury. J. Neuroeng. Rehabil. 6, 20. doi:10.1186/1743-0003-6-20

Marchal-Crespo, L., van Raai, M., Rauter, G., Wolf, P., and Riener, R. (2013). The effect of haptic guidance and visual feedback on learning a complex tennis task. Exp. Brain Res. 231, 277-291. doi:10.1007/s00221-013-3690-2

McAuley, E., Duncan, T., and Tammen, V. V. (1989). Psychometric properties of the intrinsic motivation inventory in a competitive sport setting: a confirmatory factor analysis. Res. Q. Exerc. Sport 60, 48-58. doi:10.1080/02701367.1989. 10607413

Milot, M.-H., Marchal-Crespo, L., Green, C. S., Cramer, S. C., and Reinkensmeyer, D. J. (2010). Comparison of error-amplification and haptic-guidance training techniques for learning of a timing-based motor task by healthy individuals. Exp. Brain Res. 201, 119-131. doi:10.1007/s00221-009-2014-z

Morris, D., Tan, H., Barbagli, F., Chang, T., and Salisbury, K. (2007). "Haptic feedback enhances force skill learning," in Proceedings of the Second Joint EuroHaptics Conference and Symposium on Haptic Interfaces for Virtual Environment and Teleoperator Systems (Washington, DC: IEEE Computer Society), $21-26$.

Nef, T., Guidali, M., and Riener, R. (2009). ARMin III - arm therapy exoskeleton with an ergonomic shoulder actuation. Appl. Bionics Biomech. 6, 127-142. doi: $10.1080 / 11762320902840179$

Nordin, N., Xie, S. Q., and Wünsche, B. (2014). Assessment of movement quality in robot assisted upper limb rehabilitation after stroke: a review. J. Neuroeng. Rehabil. 11, 137. doi:10.1186/1743-0003-11-137

Novak, D., Nagle, A., Keller, U., and Riener, R. (2014). Increasing motivation in robot-aided arm rehabilitation with competitive and cooperative gameplay. $J$. Neuroeng. Rehabil. 11, 64. doi:10.1186/1743-0003-11-64

Patton, J. L., Stoykov, M. E., Kovic, M., and Mussa-Ivaldi, F. A. (2006). Evaluation of robotic training forces that either enhance or reduce error in chronic hemiparetic stroke survivors. Exp. Brain Res. 168, 368-383. doi:10.1007/s00221-005-0097-8

Proteau, L. (2005). Visual afferent information dominates other sources of afferent information during mixed practice of a video-aiming task. Exp. Brain Res. 161, 441-456. doi:10.1007/s00221-004-2090-Z

Reinkensmeyer, D. J., Akoner, O., Ferris, D. P., and Gordon, K. E. (2009). Slacking by the human motor system: computational models and implications for robotic orthoses. Conf. Proc. IEEE Eng. Med. Biol. Soc. 2009, 2129-2132. doi:10.1109/ IEMBS.2009.5333978

Reinkensmeyer, D. J., and Housman, S. J. (2007). "If I can't do it once, why do it a hundred times? Connecting volition to movement success in a virtual environment motivates people to exercise the arm after stroke," in 2007 Virtual Rehabilitation (Venice), 44-48.

Reisman, D. S., Wityk, R., Silver, K., and Bastian, A. J. (2007). Locomotor adaptation on a split-belt treadmill can improve walking symmetry post-stroke. Brain 130(Pt 7), 1861-1872. doi:10.1093/brain/awm035

Rüdt, S., Moos, M., Seppey, S., Riener, R., and Marchal-Crespo, L. (2016). “Towards more efficient robotic gait training: a novel controller to modulate movement errors," in 2016 6th IEEE International Conference on Biomedical Robotics and Biomechatronics (BioRob) (Singapore), 876-881.

Ryan, R. M. (1982). Control and information in the intrapersonal sphere: an extension of cognitive evaluation theory. J. Pers. Soc. Psychol. 43, 450-461. doi:10.1037/0022-3514.43.3.450

Scheidt, R. A., Reinkensmeyer, D. J., Conditt, M. A., Rymer, W. Z., and MussaIvaldi, F. A. (2000). Persistence of motor adaptation during constrained, multijoint, arm movements. J. Neurophysiol. 84, 853-862.

Shumway-Cook, A., and Woollacott, M. H. (2007). Motor Control: Translating Research into Clinical Practice. Lippincott Williams \& Wilkins.

Wu, H. G., Miyamoto, Y. R., Gonzalez Castro, L. N., Ölveczky, B. P., and Smith, M. A. (2014). Temporal structure of motor variability is dynamically regulated and predicts motor learning ability. Nat. Neurosci. 17, 312-321. doi:10.1038/nn.3616

Conflict of Interest Statement: The authors declare that the research was conducted in the absence of any commercial or financial relationships that could be construed as a potential conflict of interest.

The reviewer, CC, and handling editor declared their shared affiliation, and the handling editor states that the process nevertheless met the standards of a fair and objective review.

Copyright (c) 2017 Marchal-Crespo, Baumann, Imobersteg, Maassen and Riener. This is an open-access article distributed under the terms of the Creative Commons Attribution License (CC BY). The use, distribution or reproduction in other forums is permitted, provided the original author (s) or licensor are credited and that the original publication in this journal is cited, in accordance with accepted academic practice. No use, distribution or reproduction is permitted which does not comply with these terms 